\title{
Longpath DOAS tomography on a motorway exhaust gas plume: numerical studies and application to data from the BAB II campaign
}

\author{
T. Laepple, V. Knab, K.-U. Mettendorf, and I. Pundt \\ Institut für Umweltphysik, Ruprecht-Karls-Universität Heidelberg, Heidelberg, Germany \\ Received: 15 March 2004 - Published in Atmos. Chem. Phys. Discuss.: 7 May 2004 \\ Revised: 28 July 2004 - Accepted: 9 August 2004 - Published: 23 August 2004
}

\begin{abstract}
This paper presents a procedure for performing and optimizing inversions for DOAS tomography and its application to measurement data. DOAS tomography is a new technique to determine 2- and 3-dimensional concentration fields of air pollutants or other trace gases by combining differential optical absorption spectroscopy (DOAS) with tomographic inversion techniques. Due to the limited amount of measured data, the resulting concentration fields are sensitive to the inversion process. Therefore detailed error estimations are needed to determine the quality of the reconstruction. In this paper we compare different row acting methods for the inversion, present a procedure for optimizing the parameters of the reconstruction process and propose a way to estimate the error-fields by numerical studies. The procedure was applied to data from the motorway emission campaign BAB II. Two dimensional $\mathrm{NO}_{2}$ cross sections at right angles to the motorway could be reconstructed qualitatively well at different meteorological situations. Additionally we present error fields for the reconstructions which show the problems and skills of the used measurement setup. Numerical studies on an improved setup for future motorway campaigns show, that DOAS tomography is able to produce high quality concentration maps.
\end{abstract}

\section{Introduction}

The measurement of trace gas concentration distributions in the atmosphere is an important tool for quantifying atmospheric emissions, chemistry and transport. It can contribute to the validation of chemical transport models (CTM), the improvement of emission inventories or for emission monitoring (e.g. leakages in industrial installations).

Correspondence to: T. Laepple

(thomas.laepple@iup.uni-heidelberg.de)

\subsection{Tomography for mapping trace gas distributions}

Two and three dimensional concentration distributions of trace gases can be obtained by combining path-integrating measurement techniques along a large number of light paths with tomographical inversion techniques. In comparison point sampling techniques can only give local details of the concentration maps and conclusions to larger scales may be falsified by small scale fluctuations. Tomographic lineintegrating measurement techniques for trace gases were proposed by Wolfe (1980) and first indoor measurements were realized by Yost et al. (1994) using open-path Fourier infrared absorption spectroscopy. Since then there have been improvements in speed and spatial resolution (e.g. Drescher et al., 1996; Fischer et al., 2001), but to our knowledge all experiments have been restricted to the laboratory environment so far.

\subsection{DOAS tomography}

Differential optical absorption spectroscopy (DOAS) (e.g. Platt, 1994; Perner et al., 1976) is a path-integrating measurement technique for trace gases like ozone, nitrogen oxides, $\mathrm{SO}_{2}$, halogen oxides ( $\left.\mathrm{BrO}, \mathrm{IO}, \mathrm{OClO}\right)$ and many hydrocarbons. In a typical so-called long-path (LP) setup a beam of white light, emitted by a telescope, travels through the atmosphere to a retro-reflector and back to the telescope. Absorption patterns in the recaptured light allow to determine the column density (the integrated concentration) of the trace gases along the light path. One advantage of DOAS is the simultaneous measurement of several trace gases (e.g. $\mathrm{NO}_{2}$, $\mathrm{SO}_{2}, \mathrm{HCHO}, \mathrm{HONO}$, and ozone in the UV region between 280 and $380 \mathrm{~nm}$ ) which allows detailed direct conclusions on the air chemistry. LP-DOAS was already used to produce one-dimensional trace gas profiles by placing retroreflectors on balloons (e.g. Veitel et al., 2002) or on a mountain at different heights (Platt, 1978). First outdoor DOAS tomography 
experiments were carried out during the motorway campaign BAB II in 2001, which was organised by Fiedler et al. (2001). More details are given by Pundt et al. (2004), Knab (2003) and in Sect. 2.

In comparison to other tomographic applications like in medicine, DOAS-tomography has only a very limited number (10-100) of well known light paths. Therefore the reconstruction technique is not time critical but attention has to be paid on the a-priori information added to the ill-posed problem, implicitly by the algorithm or explicitly by the selected parameters. This requires extensive studies on the reliability of the reconstructed concentration fields. These studies are a main subject of this paper.

\subsection{Overview over inversion techniques}

Due to the limited amount of data in DOAS-tomography a discretization is needed which describes the concentration field by a finite number of parameters accepting losses in accuracy.

Drescher et al. (1996) proposed an inversion algorithm called smooth basis function minimization technique (SBFM) for the reconstruction of indoor trace gas measurements. The concentration field is parameterized nonlinearly as a sum of several Gaussians with some free parameters. A global optimization algorithm is used to determine the set of parameters fitting the measurement data best. While SBFM is reported to be suitable for indoor measurements examining the mixing of trace gas from located sources (Fischer et al., 2001), it could not be applied successfully in our case of a motorway exhaust plume. The plume shapes for the motorway campaign estimated by a chemical transport model (D. Bäumer, personal communication) could not be described by Gaussians with a number of parameters determinable by the measurement data. Sharp concentration slopes are appearing near emission sources, if the wind is blowing from one specific direction. This is not the case in indoor situations. Other parameterizations for the use at the motorway situation involved an unquantifiable amount of a priori information. One main problem is the difficulty to determine the quality of the reconstruction due to the nonlinearity of the inversion and the long computation time for the global optimization (in the order of hours for our problem size) which does not allow extensive numerical sensitivity studies.

The more common approach is to discretize the continuous concentration field by a linear combination of a finite number of basis functions. The advantage of this linear approach is that the resulting discrete linear inversion problem is better to handle and does not require the knowledge of the algebraic form of the concentration field. (The nonlinear approach SBFM only works well if the concentration field can be described by superposition of few Gaussians). Therefore we use the linear approach. In the literature many methods can be found to solve this linear inversion problem (e.g. Groetsch, 1993).
In environmental science a popular class of methods are iterative row-acting-methods (RAM). They were often used to deal with large size problems because they only act on the rows of a matrix and save memory space. Their simplicity and their good regularization and smoothing characteristics still makes them interesting and they are successfully applied on tomographic problems (e.g. Kak and Slanley, 1988). In environmental sciences, Ziemann et al. (2001) use the simultaneous iterative reconstruction technique (SIRT) a member of this algorithm class in acoustic tomography for determining small scale land surface characteristics. Todd and Ramachandran (1994) use row acting methods for numerical studies on FTIR-tomography. The disadvantage of these techniques is that the a priori information which is always needed to solve the ill-posed inversion problem is not added explicitly but is included in the nature of the algorithm, the first guess and the number of iterations.

There are inversion techniques which include the a priori information explicitly. Application of a statistical approach to atmospheric remote sensing can be found in Rodgers (2000). A constrained optimization method - where a smoothness function is used as quadratic constraint - is used successfully by Fehmers et al. (1998) in the field of the tomography of the ionosphere. If good a priori information is available these inversion techniques are useful to include such information. Further work has to be done to investigate these techniques and to investigate which a priori information can be integrated depending on the problem.

Due to their regular use in tomographic problems similar to our problem we decided to use row acting methods as a starting point for the new tomographic DOAS technique and the study described here. First we describe the discretization process and discuss different basis functions and different row acting methods in Sect. 3. In contrast to other studies we try to investigate the complete characteristics of the chosen RAM's by numerical studies in Sect. 5 and choose the best parameter set for our reconstruction. In Sect. 6 we apply this optimized algorithm on the motorway data.

Independent of the inversion technique it is important to quantify the quality of reconstructions. To our knowledge the absolute reconstruction error has not yet been estimated in tomographic applications in atmospheric sciences. Drescher et al. (1997) compared reconstructed concentration fields with real point measurements. Todd and Ramachandran (1994) performed numerical experiments judging the reconstruction quality by some quality criteria, but only considering concentration fields constructed as sum of several randomly located Gaussians and ignoring the errors caused by the discretization. Price et al. (2001) mentioned errors due to measuring the light paths sequentially but did not quantify them.

In Sect. 4 we analyse the types of occuring errors and estimate them in Sect. 6 for the motorway campaign. For further reduction of uncertainties in the results of the motorway campaign we propose in Sect. 6.6 an improved measurement setup for future campaigns. 

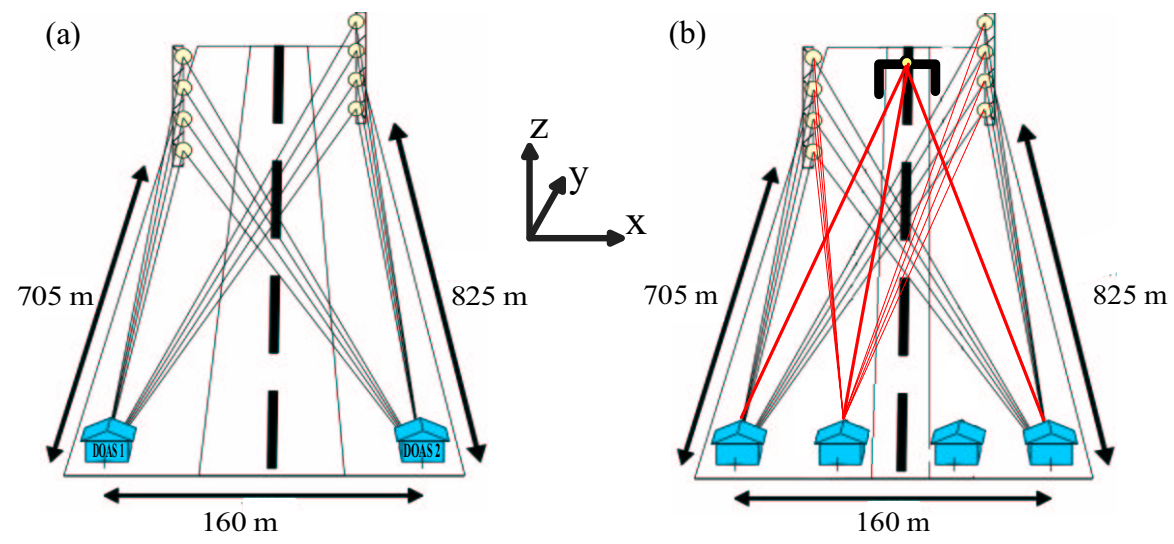

Fig. 1. DOAS tomography setups for the measurements of cross sections of vehicle exhaust gas plumes at right angles to the motorway. Setup (a) was used during the motorway campaign BAB II. Two DOAS telescopes were placed on opposite sides of the motorway. 4 retroreflectors were mounted on two cranes in about $800 \mathrm{~m}$ distance. With the stepping technique 16 lightbeams were realized, $8 \mathrm{crossing}$ and 8 going parallel to the carriageway. The enhanced setup (b), desribed in Sect. 6.7, consists of 4 telescopes and 9 retroreflectors. It is able to measure the cross sections with much better accuracy. For better clarity only the lightbeams of the telescopes on the left hand side are drawn in completely.

\section{DOAS tomography measurements at a motorway}

During the field campaign BAB II (Experimental determination of emissions from motor vehicle traffic on motorways and comparison to calculated emissions) at the motorway A 656 in Germany, organized by the Institute for Meteorology and Climate Research (IMK) of the Forschungszentrum Karlsruhe, first Longpath DOAS tomography measurements took place in April, May 2001. It was the first pathintegrating tomographic 2-D outdoor measurement of trace gases. For details on the campaign please refer to Fiedler et al. (2001). The DOAS tomography measurements are described by Knab (2003) and Pundt et al. (2004).

A setup of 16 light paths was realized by using two conventional long-path DOAS systems and directing them successively towards eight retro-reflector arrays. The environmental conditions for the time period studied here were chosen deliberately: During that time there were both, a relatively continuous vehicle flux and undisturbed air crossing the motorway. Thus the situation was assumed to be homogeneous in the direction of the carriageway and a concentration cross section at right angles to the motorway could be derived. The measurement setup is shown on the left part of Fig. 1.

The motorway induced turbulences and chemical transformations at the measurement site which had been examined by Vogel et al. (2000) and Bäumer (2003) with the mesoscale chemistry transport model system KAMM/ DRAIS. A $\mathrm{NO}_{2}$ concentration field generated with this model (D. Bäumer, personal communication) was used for numerical experiments in this study and will, in the following, just be referred to as CTM BAB II plume (see Fig. 4a).

\section{Tomography}

The distribution of a trace gas in the atmosphere is usually described as a continuous concentration field $c(\mathbf{r})$. Assuming the concentration field is known, a DOAS measurement along a light path $\mathrm{LP}_{i}$ gives the data (slant column density)

$d_{i}=\int_{L P_{i}} \operatorname{drc}(\mathbf{r})$.

This is the forward model and is well known in our case. The unit of the data used here is $\mathrm{ppb} * \mathrm{~m}$ which corresponds to the integrated concentration over the light path.

\subsection{Discretization}

A model is needed which describes the continuous concentration field with a finite number of parameters. We will call this a discrete state model. If there are $\mathrm{m}$ lightbeams resulting in $\mathrm{m}$ values of measurement data $d_{1}$ to $d_{m}$, they are assembled to a data vector $\boldsymbol{d}$ and the $\mathrm{n}$ parameters $s_{1}$ to $s_{n}$ describing the state model concentration field in a state vector $s$.

Data vector $\boldsymbol{d}=\left(d_{1}, d_{2}, \ldots, d_{m}\right)^{t} \in D$

State vector $\boldsymbol{s}=\left(s_{1}, s_{2}, \ldots, s_{n}\right)^{t} \in S$

The according vector spaces are called data (vector) space $D$ and state (vector) space $S$. A discrete state model can be realized by a family of $\mathrm{n}$ basis functions $\left(b_{j}\right)_{j \in\{1 . . n\}}$. Then the model concentration field $c(\boldsymbol{s}, \mathbf{r})$ is a linear combination of the basis functions $b_{j}(\mathbf{r})$ with the state vector components $s_{j}$ as coefficients.

$c(\boldsymbol{s}, \mathbf{r})=\sum s_{j} b_{j}(\mathbf{r})=\boldsymbol{s} \cdot \mathbf{b}(\mathbf{r})$ 
(a)

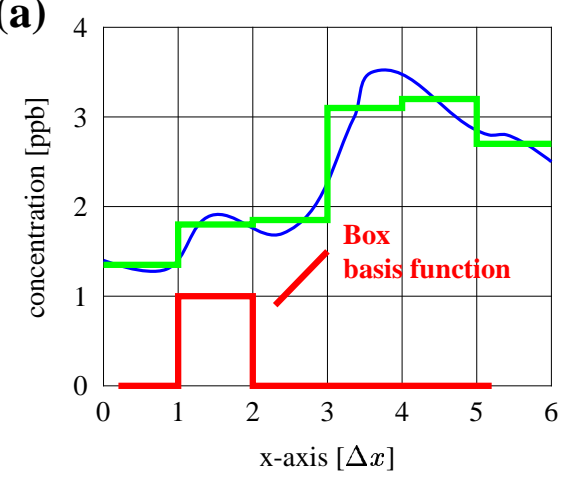

(b)

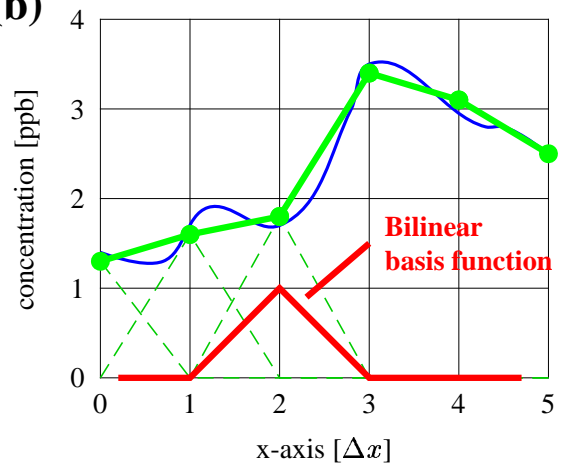

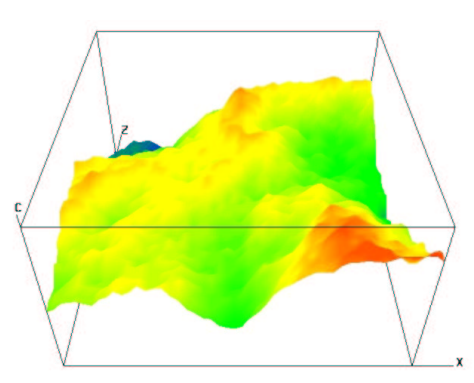

(c) original

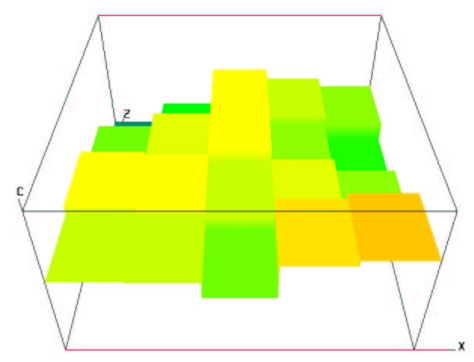

(d) $5 \times 4$ box model

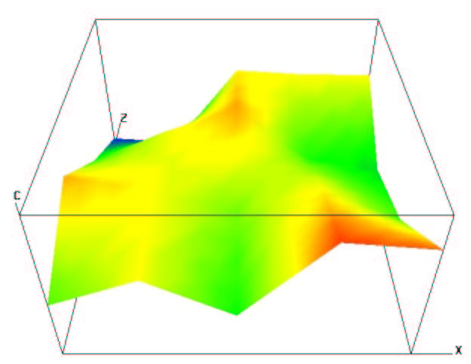

(e) $5 \times 4$ bil. int model

Fig. 2. Discretization models. The upper two subfigures show a 1-dimensional cross section of a concentration field. In the case of the box model (a) the real concentration field (blue) is approximated by a step function (green), which is constant within each box. For the bilinear interpolation model (b) the basis functions (red) are pyramid shaped. They have a peak of height one above the affiliated lattice point and reach zero at the neighboring lattice points. The resulting model function (green) is continuous and interpolates the values at the lattice points linearly. In subfigures (c), (d) and (e) the discrete modeling of a continuous 2-D function is visualized in color contour plots: A continuous concentration field (c), its best approximation with the $5 \times 4$ box model (d) and the $5 \times 4$ bilinear interpolation model (e).

The basis functions have to be linearly independent. Then for each continuous concentration field $c(\mathbf{r})$ a unique state vector $\boldsymbol{s}$ exists that minimizes the misfit $\|c(\mathbf{r})-\boldsymbol{s} \cdot \mathbf{r}\|$. In the following we assume the concentration to be invariant of the z-axis. This refers to the reconstruction of 2-dimensional cross sections of 3-dimensional trace gas fields. We investigate two kinds of discrete state models: the box model and the bilinear interpolation model.

\subsubsection{Box model}

For the box or pixel model (e.g. Kak and Slanley, 1988) the area of interest is divided into (usually rectangular) boxes. Each box corresponds to a basis function

$b_{j}(\mathbf{r})= \begin{cases}1 & \text { if } \mathbf{r} \in \text { box } \mathbf{j} \\ 0 & \text { else }\end{cases}$
The model concentration fields thus are step functions which are constant within the boxes.

$c(\boldsymbol{s}, \mathbf{r})=\left\{\begin{array}{llll}s_{1} & \text { if } & \mathbf{r} & \text { in box 1 } \\ s_{2} & \text { if } & \mathbf{r} & \text { in box 2 } \\ \cdot & & & \\ \cdot & & & \end{array}\right.$

Here the state vector components are the model concentrations of the different boxes. Figure 2a shows a representation of a one-dimensional model field by such box functions. The concentration field $c(\mathbf{r})$ is approximated best, if the height of the step $s_{j}$ is the average concentration in the referring box: $s_{j}=\langle c(\mathbf{r})\rangle_{\text {box }_{\mathrm{j}}}$.

\subsubsection{Bilinear interpolation model}

For the bilinear interpolation model (e.g. Ingesson et al., 1998) a point lattice is laid over the area of interest. Each 
lattice point $\mathbf{r}_{j}=\left(\mathrm{x}_{j}, \mathrm{y}_{j}\right)$ refers to a bilinear basis function $b_{j}$ :

$$
\begin{aligned}
& b_{j}=t_{x j}(x) t_{y j}(y) \\
& t_{x j}= \begin{cases}1-\frac{1}{\Delta x}\left|x-x_{j}\right| \text { if }\left|x-x_{j}\right| \leq \Delta x \\
0 & \text { else }\end{cases} \\
& t_{y j}=\text { analog }
\end{aligned}
$$

where $\Delta \mathrm{x}$ is the lattice width in $\mathrm{x}$-direction. The basis functions are pyramid-shaped and have a peak of height 1 above the affiliated lattice point. The value of the modeled concentration field at the lattice points is given by the components of the state vector

$c\left(s, \mathbf{r}_{j}\right)=s_{j} \quad$ for all lattice points $\mathbf{r}_{j}$.

At the other points the model field is determined by bilinear interpolation of the values at the four neighboring lattice points. Figure $2 b$ shows a representation of a onedimensional model field by such bilinear basis functions.

Due to the continuity of the bilinear basis function they can describe a continuous concentration field better than the discontinuous box-basis functions. Therefore they are more appropriate in our case. In Fig. 2c and e, this is demonstrated by modeling a test concentration field using the two types of basis functions. The advantage of bilinear basis functions will also be confirmed in Sect. 5, where we compare the reconstruction quality, using the box and the bilinear representation in turns.

\subsubsection{Resolution of the discretization}

The state model has to describe the real concentration field as accurately as possible with a small number of basis functions $\mathrm{n}$.

If the chosen resolution is not fine enough (too small dimension of the state vector) the difference between the continuous concentration field and the best discrete approximation leads to errors due to the discretization, so-called "discretization errors" in the reconstruction. If the resolution is too fine, the problem gets highly underdetermined and more a priori information is needed to solve the inversion problem. (A further discussion of these errors can be found in Sect. 4.) The best resolution is dependent on the information content of the measurements and the a-priori information available and will be determined in numerical studies in Sect. 5 .

\subsection{Discrete linear inverse problem}

Approximating the real concentration field by the model field, the forward model (1) becomes $\boldsymbol{d}=\mathbf{F} s$ with forward matrix:

$$
f_{i j}=\int_{L P_{i}} d r b_{j}(\mathbf{r})
$$

In the case of the box model the entry $f_{i j}$ of the forward matrix corresponds to the length of light path i through box j. The problem of solving Eq. (9) for a given data vector $\boldsymbol{d}$ is a discrete linear inverse problem.

This problem is in general ill-posed: If Eq. (9) is over- or mixed-determined no exact solution exists, but only an approximate solution. If Eq. (9) is mixed- or under-determined the solution is not unique. If the condition number (e.g. the ratio of largest to smallest singular value of the matrix) of $F$ is large, the solution is not stable, i.e. it is sensitive to small errors in the data.

The first problem can be overcome by using the approximate minimum misfit solution (least squares solution) as physical solution. The latter two problems can only be remedied by adding additional a priori information, e.g. the information, that the concentration field either is positive allover or that it fulfills certain smoothness conditions. Often the minimum norm solution is demanded, but the a priori information to favor the state with the smallest norm is physically not reasonable in our case. The approximate minimum norm minimum misfit solution is also known as generalized inverse, and can be calculated by singular value composition (SVD) (e.g. Groetsch, 1993).

To take the measurement error on the data into account, a weighting matrix $\mathbf{W}$ can be introduced which is the inverse of the covariance matrix. If the noise on the data can be assumed to be uncorrelated $\mathbf{W}$ is given by the elements $W_{i i}=1 / \varepsilon_{i}^{2}$. The weighting is applied by the substitution:

$$
\tilde{d} \longleftrightarrow \mathbf{W}^{1 / 2} \boldsymbol{d}, \quad \tilde{\mathbf{F}} \longleftrightarrow \mathbf{W}^{1 / 2} \mathbf{F} \quad \text { and } \quad \tilde{s} \longleftrightarrow s
$$

This is assumed to be already done in the following discussion.

\subsection{Row acting methods}

As mentioned in the introduction we use row acting methods (RAM) in this study to solve the discrete linear inverse problem.

RAM are a class of iterative algorithms to solve linear systems of equations - especially tomographic discrete linear inverse problems. The common names of row acting methods like Algebraic Reconstruction Technique (ART) (Herman et al., 1973) combine an inversion technique (i.e. the technique for solving the system of Eq. 9) with a special discretization model (e.g. box, bilinear). As we want to use the algorithm independently from the discretization model, we use the separate names "ClassicalName-like" to allow a better comparison. (Our "ART-like" algorithm for example is the algorithm ART from the literature but independent of the discretization model.)

\subsubsection{The ART-like method}

The sequential iterative projection method or Kaczmarz method (Kaczmarz, 1937) was firstly used in tomography 


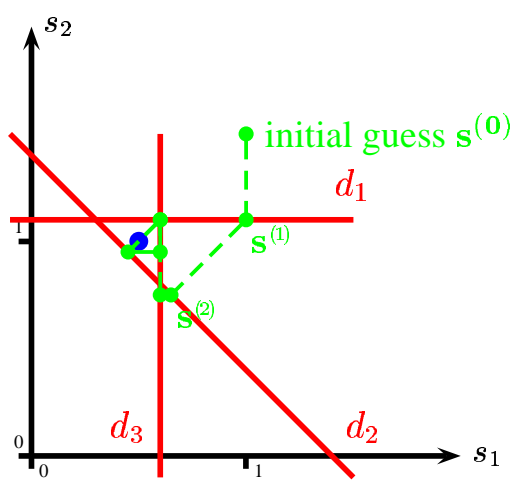

(a) sequential RAM (ART-like)

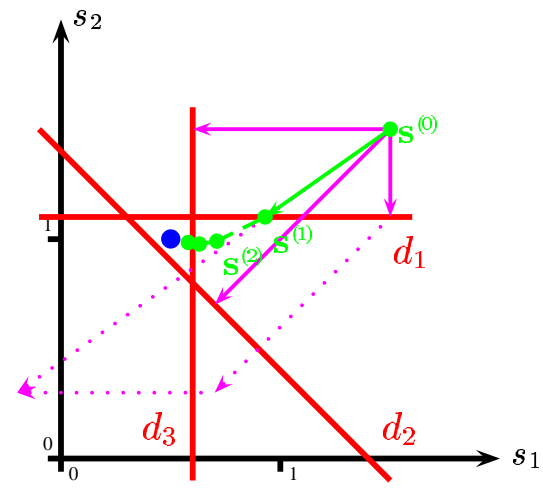

(b) simultaneous RAM (SIRT-like)

Fig. 3. Row acting methods (RAM) graphically. Each data point di refers to a hyperplane in state space (red lines). If an exact solution exists, it must lie on all these hyperplanes. Here the inverse problem is over-determined, and there is no exact solution (there is no intersection for all three hyperplanes). Starting point is the initial guess s ${ }^{(0)}$. For the sequential RAM (a) the actual guess is iteratively projected onto one hyperplane after another. Because of the over-determination the iteration sequence $\left(\mathrm{s}^{(j)}\right)$ (green) oscillates cyclically convergent in the neighborhood of the intersections of the hyperplanes. For the simultaneous RAM (b) the hypothetical changes due to projections onto all hyperplanes (pink vectors) are gathered first. Then their average (green vector) is added to the actual guess. Here the state vector sequence $\left(\mathrm{s}^{(j)}\right)$ approaches the real state slowly but consequently.

in combination with box basis functions under the name algebraic reconstruction technique. It is based on the following idea: Each row $\mathbf{f}_{i} \cdot s=d_{i}$ of matrix Eq. (9) refers to one data-point and fixes a (n-1) dimensional hyperplane in a ndimensional state space, on which any existing solution must lie. This is shown graphically in Fig. 3a for one dimensional hyperplanes in a two dimensional space. If a solution exists, it is thus situated on the intersection of all $n$ hyperplanes. The algorithm starts with an initial guess $\mathbf{s}^{(0)}$. The solution is determined by iteratively projecting the actual guess onto a hyperplane and taking the result as new actual guess.

$\mathbf{s}^{(k+1)}=\mathbf{s}^{(k)}+\alpha\left(\frac{d_{i}}{\left\|\mathbf{f}_{i}\right\|}-\mathbf{s}^{(k)} \frac{\mathbf{f}_{i}}{\left\|\mathbf{f}_{i}\right\|}\right) \frac{\mathbf{f}_{i}}{\left\|\mathbf{f}_{i}\right\|}$,

where $\mathrm{i}=(\mathrm{k} \bmod \mathrm{m})+1$.

Here $\mathrm{k}$ is the iteration number and $\mathrm{i}$ is the number of the hyperplane onto which $\mathbf{s}^{(k)}$ is projected in the actual iteration step. The damping parameter $\alpha$ is 1 for the basis algorithm. The optimal termination of the iteration can be determined in numerical experiments.

If a unique solution exists, the iteration sequence $\left(\mathbf{s}^{(k)}\right)_{k \in N}$ converges to this solution. If the system of equations is underdetermined, $\left(\mathbf{s}^{(k)}\right)$ converges to the solution closest to the initial guess in the sense of the Euclidian vector norm. If the system of equations is mixed- or over-determined and the data vector is "noisy", generally no solution exists. Then ART is cyclically convergent, i.e. after a lot of iteration the sequence $\left(\mathbf{s}^{(k)}\right)$ follows to a fixed closed trajectory of period $\mathrm{m}$ (e.g. Censor et al., 1983). In Fig. 3a this is the case because this example is overdetermined and has no exact solution (three hyperplanes in a two dimensional state space).
In such cases a better convergence can be obtained by using a damping parameter $\alpha$ decreasing from 1 to 0 in the course of the iteration. If the concentration field is zero at some locations, the convergence can be ameliorated, by setting negative values back to zero in each pass. For the motorway situation it would not have made any sense to use this technique. The row acting methods looked at here are implicitly smoothing the solution if the iteration is terminated prematurely. An optimum iteration number can be found by surveying the Iteration process by eye, by guessing roughly or as done in this study by numerical experiments.

\subsubsection{The SIRT-like method}

The combination of the simultaneous iterative projection method and a box discretization model is known as simultaneous iterative reconstruction technique (SIRT) (Kak and Slanley, 1988).

This method differs from the sequential iterative projection method, by the fact that the correction of a state due to the projection is not immediately applied. Instead, before making any changes to $\mathrm{s}$ all $\mathrm{m}$ equations are gone through calculating the hypothetical change due to the projection onto the hyperplane. At the end of one pass the average over all these hypothetical changes is taken and applied to the state vector:

$\mathbf{s}^{(k+1)}=\mathbf{s}^{(k)}+\frac{\alpha}{m} \sum_{i=1}^{m}\left(\frac{d_{i}}{\left\|\mathbf{f}_{i}\right\|}-\mathbf{s}^{(k)} \cdot \frac{\mathbf{f}_{i}}{\left\|\mathbf{f}_{i}\right\|}\right) \frac{\mathbf{f}_{i}}{\left\|\mathbf{f}_{i}\right\|}$

Increasing the iteration number $\mathrm{k}$ by 1 in this case signifies a whole pass through all equations. The state vector sequence converges to a minimum-misfit-solution closest to the initial 
guess (Van der Sluis and van der Vorst, 1987). This is shown in Fig. $3 b$ which is based on the same equation system as in Fig. 3a but with the SIRT algorithm. The three hypothetical changes for the first step are explicitly plotted. For the other steps only the applied change to the state vector are shown. In contrast to Fig. 3a, the state vector converges to the minimum misfit solution.

\subsubsection{The SART-like inversion method}

Another modification is an algorithm known - in combination with bilinear basis functions - as simultaneous algebraic reconstruction projection method (SART)(Kak and Slanley, 1988).

The projections applied simultaneously as in the simultaneous iterative projection method, but the weights of the corrections are different.

$\mathbf{s}_{j}^{(k+1)}=\mathbf{s}_{j}^{(k)}+\frac{1}{\sum_{i=1}^{m} f_{i j}} \sum_{i=1}^{m} \frac{\left(d_{i}-\mathbf{s}^{(k)} \cdot \mathbf{f}_{i}\right) f_{i j}}{\sum_{\alpha=1}^{n} f_{i \alpha}}$

Here the iteration sequence converges to a minimizer of a weighted least square functional from any initial guess (Jiang and Wang, 2001). The convergence is faster than that of the SIRT-like inversion method. In DOAS-tomography applications (state vector size 10-100) the computation time for the row acting methods is about 1000-10000 iterations per second on a $1 \mathrm{GHZ}$ Pentium processor. Therefore the convergence speed is not important for our applications.

\section{Error estimation}

The reconstruction error field $\Delta c(\mathbf{r})$ is the difference between the concentration field reconstructed by a DOAStomography measurement $c_{r e c}(\mathbf{r})$ and the real concentration field $c_{\text {real }}(\mathbf{r})$.

$\Delta c(\mathbf{r})=c_{\text {real }}(\mathbf{r})-c_{\text {rec }}(\mathbf{r})$

For simplicity we will also call continuous test-fields $c_{\text {real }}(\mathbf{r})$ which are used in numerical studies.

In this section we describe the sources of the reconstruction error and a way to estimate them. The practical procedures for optimizing the reconstruction process with respect to a low reconstruction error and for estimating the error for the motorway campaign are presented in the referring Sects. 5 and 6.

\subsection{Sources of the reconstruction error}

The reconstruction error has four causes:

1. the measurement error,

2. the discretization error in the data,

3. the discretization error in the state,
4. the inversion error.

For a better understanding we introduce the following operator notation:

- $\mathcal{D}$ Discretization operator; maps a concentration field $c(\mathbf{r})$ to the best approximation state vector $\boldsymbol{s}=\mathcal{D} c(\mathbf{r})$.

- $\mathcal{D}^{\dagger}$ Continuization' operator; leads from the state vector $\mathrm{s}$ to the affiliated concentration field $c(\boldsymbol{s}, \mathbf{r})=\mathcal{D}^{\dagger} \boldsymbol{s}$.

- $\mathcal{F}$ Forward operator; forward models data from the discrete state vector $\boldsymbol{d}=\mathcal{F} \boldsymbol{s}$ (see Eq. 9) .

$-\mathcal{G}$ Continuous forward operator; forward models data from a continuous concentration field $\boldsymbol{d}=\mathcal{G} c(\mathbf{r})$ (see Eq. 1).

$-\mathcal{F}^{\dagger}$ Inversion Operator; describes the application of the inversion method to the data $s=\mathcal{F}^{\dagger} \boldsymbol{d}$.

The "continuization" operator $\mathcal{D}^{\dagger}$ is a pseudo-inverse to the discretization operator $\mathcal{D}$. The inversion operator $\mathcal{F}^{\dagger}$ is pseudo-inverse to the discrete forward modeling operator $\mathcal{F}$.

In this notation the reconstruction and the simulation of a measurement and reconstruction are:

Reconstruction $\quad c_{\text {rec }}(\mathbf{r})=\mathcal{D}^{\dagger} \mathcal{F}^{\dagger} \boldsymbol{d}$

Simulation $\quad c_{\text {rec }}(\mathbf{r})=\mathcal{D}^{\dagger} \mathcal{F}^{\dagger} \mathcal{G} c(\mathbf{r})$

The measurement error $\Delta \boldsymbol{d}_{\text {meas }}$ is the difference between the measured data and the data that would be obtained from an ideal experiment (i.e. data forward modeled from the real, continuous field). $\Delta \boldsymbol{d}_{\text {meas }}$ consists of the error in the individual measurement devices and the so called stepping error, which occurs if the different light paths are not measured simultaneously. It is propagated through the inversion the same way as the data (because of the linearity of the two operators) yielding the propagated measurement error:

$\Delta c_{\text {meas }}(\mathbf{r})=\mathcal{D}^{\dagger} \mathcal{F}^{\dagger} \Delta \boldsymbol{d}_{\text {meas }}$

The discretization leads to errors in two ways: Firstly, an arbitrary continuous concentration field generally cannot be exactly approximated by the discretized field: $\mathcal{D}^{\dagger} \mathcal{D} \neq 1$.

Secondly, the forward modeling of measurement data from the discretized field differs from the data obtained from the real, continuous field: $\mathcal{F} \mathcal{D} \neq \mathcal{G}$.

If the inverse problem (9) does not have a unique solution, i.e. because it is ill-posed, a priori information has to be employed to select a solution. The inversion error arises, because normally the a priori information is not completely correct. $\mathcal{F}^{\dagger} \mathcal{F} \neq 1$.

We get the sum of discretization errors and the inversion error in the concentration as

$\Delta c_{d i}(\mathbf{r})=\left(1-\mathcal{D}^{\dagger} \mathcal{F}^{\dagger} \mathcal{G}\right) c_{\text {real }}(\mathbf{r})$ 
which corresponds to simulating a perfect measurement on a concentration field, reconstructing the field and comparing it to the original concentration field.

If all operators are linear - as it is the case for linear discretization models and row acting methods (each projection step is linear and thus also the whole inversion), then the total reconstruction error is the sum of Eqs. (17) and (18):

$\Delta c(\mathbf{r})=\Delta c_{\text {meas }}(\mathbf{r})+\Delta c_{d i}(\mathbf{r})$

Therefore the two parts can be treated separately which enhances the computational speed of the error estimation and the knowledge about the error causes.

Usually the resolution matrix $\mathbf{R}=\mathbf{F}^{\dagger} \mathbf{F}$ is introduced to investigate the reconstruction quality. Using this matrix the inversion error can be determined as $(1-\mathbf{R}) s_{\text {real }}$ neglecting the discretization error. As we work with a moderate resolution, this disregarding is not acceptable in our case.

\subsection{Estimation of the error fields}

For the error estimation the error fields $\Delta c_{\text {meas }}(\mathbf{r})$ and $\Delta c_{d i}(\mathbf{r})$ are considered as random functions and the aim is to determine their random distribution (for simplicity, we use the same notation for the random function and it's realization). In order to get the total reconstruction error $\Delta c(\mathbf{r})$ we have to convolute the two random distributions at the end.

The measurement error $\Delta \boldsymbol{d}_{\text {meas }}$ is assumed to be a Gaussian distributed random vector of mean zero. This means the propagated measurement error $\Delta c_{\text {meas }}(\mathbf{r})$ is also Gaussian distributed and of mean zero. Assumed covd $\boldsymbol{d}_{\text {meas }}$ is the covariance of the $\Delta \boldsymbol{d}_{\text {meas }}$ and the continuous fields are discretized on a finite grid, then the covariance of the propagated measurement error is

$\operatorname{covc}_{\text {meas }}=\left(\mathcal{D}^{\dagger} \mathcal{F}^{\dagger}\right)\left(\operatorname{covd} \boldsymbol{d}_{\text {meas }}\right)\left(\mathcal{D}^{\dagger} \mathcal{F}^{\dagger}\right)^{\dagger}$.

The operator $\mathcal{D}^{\dagger} \mathcal{F}^{\dagger}$ can be determined by applying the inversion method on the $\mathrm{m}$ data basis vectors.

The discretization and inversion error $\Delta c_{d i}(\mathbf{r})$ depends on the shape of the field to be reconstructed. Consequently, a priori information is needed for determining $\Delta c_{d i}(\mathbf{r})$. Without any a priori information on the concentration field, the reconstruction error would be infinitely high. Imagine a DOAS tomography setup and a concentration field with a very steep and high peak in the gap between some light paths. Such peak is not detectable with the given setup. Only with information about smoothness such a field can be excluded. The concentration field to be reconstructed is a random function $c_{\text {real }}(\mathbf{r})$ and the prior information is represented by its probability distribution. Applying Eq. (18) on it leads to the probability distribution of the derived random function $\Delta c_{d i}(\mathbf{r})$. In practice the probability distribution of $c_{\text {real }}(\mathbf{r})$ was realized by generating a set of test fields $\left(c_{k}(\mathbf{r})\right)_{k \in N}$ all of which are assumed to have the same probability. Applying Eq. (18) to all of these test fields produces a set of reconstruction error fields $\left(\Delta c_{d i_{k}}(\mathbf{r})\right)_{k \in N}$ which can be evaluated statistically.

\subsection{Reconstruction quality criteria}

In simulations and validation experiments the reconstructed concentration field can be compared with a "real" field. Quality criteria are needed, which summarize the overall reconstruction quality in a single figure. They can be used in numerical simulations to determine optimum reconstruction techniques and parameters. Apart from the criterion "nearness", which has been used in former studies (e.g. Todd and Ramachandran, 1994), we suggest here two further criteria, the "normalized maximum difference" and the "normalized average difference". The choice of the criterion depends on the further use of the reconstruction result. All of the proposed criteria are calculated from the reconstruction error $\Delta c(\mathbf{r})=c_{\text {real }}(\mathbf{r})-c_{\text {rec }}(\mathbf{r})$ and the "real" field $c_{\text {real }}(\mathbf{r})$.

\subsubsection{Nearness}

The quality criterion nearness is a normalized 2-norm (Euclidian norm) of the reconstruction error:

$$
\begin{aligned}
\text { Nearness } & =\frac{1}{N}\|\Delta c\|_{2} \\
& =\frac{1}{N} \sqrt{\int d r^{2}\left(c_{\text {rec }}(\mathbf{r})-c_{\text {real }}(\mathbf{r})\right)^{2}} \\
\text { where } \mathrm{N} & =\sqrt{\int d r^{2}\left(c_{\text {real }}(\mathbf{r})-\overline{c_{\text {real }}(\mathbf{r})}\right)^{2}} .
\end{aligned}
$$

Normalization makes the nearness invariant to the multiplication of the concentration field by a scalar factor and to the addition of a constant function. If reconstruction techniques are compared for a whole set of test fields, the normalization is necessary, because it makes the nearness comparable for plumes of different sizes. The nearness has the following meaning:

NEARNESS $=0-$ ideal agreement

NEARNESS $=1-$ same agreement as constant average field

The quality criteria nearness should be used, if the overall shape of the concentration field is of interest. Our definition of nearness is based on a similar definition used by Todd (e.g. Todd and Ramachandran, 1994) with reference to Herman et al. (1973) and Herman and Rowland (1973). Todd calculated the nearness on the discrete state vector and thus ignored the discretization error. Drescher et al. (1997) used a very similar discrete quality criterion called "figure of merit" by setting the normalization factor to $\mathrm{N}=\left\|s_{\text {real }}\right\|$ and thus renouncing the compensation of shifting. 


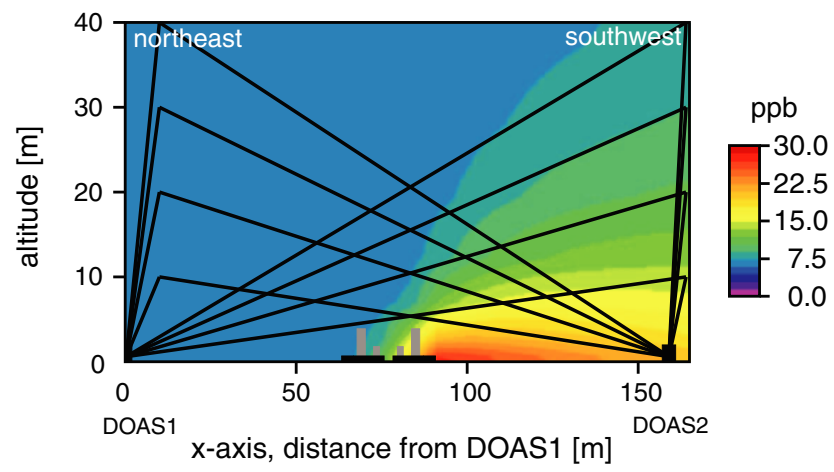

(a) Original CTM BAB II plume
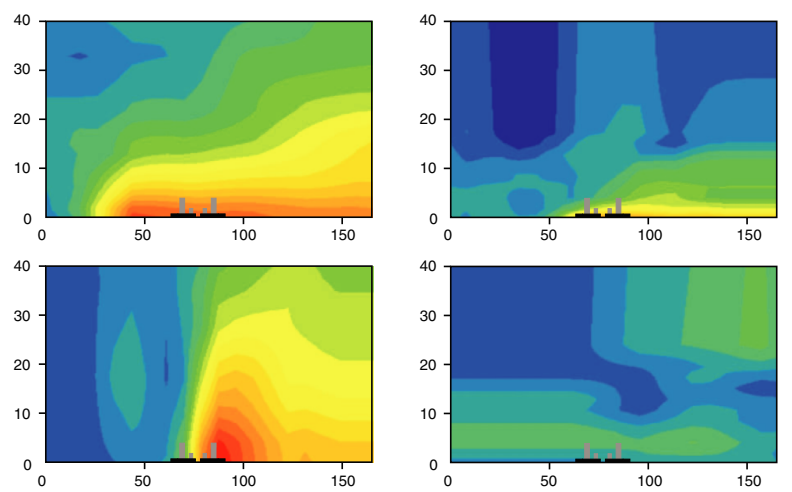

(b) Testplumes

Fig. 4. CTM BAB II plume and examples of derived test fields. (a) The $\mathrm{NO}_{2}$ concentration field, produced by D. Bäumer (personal communication, see Sect. 1.5), for the BAB II situation with a chemistry transport model. It was used in this study for numerical experiments: (b) A set of test fields was generated by squeezing the model plume in intensity, squeezing and shifting it in space and adding small scale random field. Four realizations of this modification procedure are shown in (b).

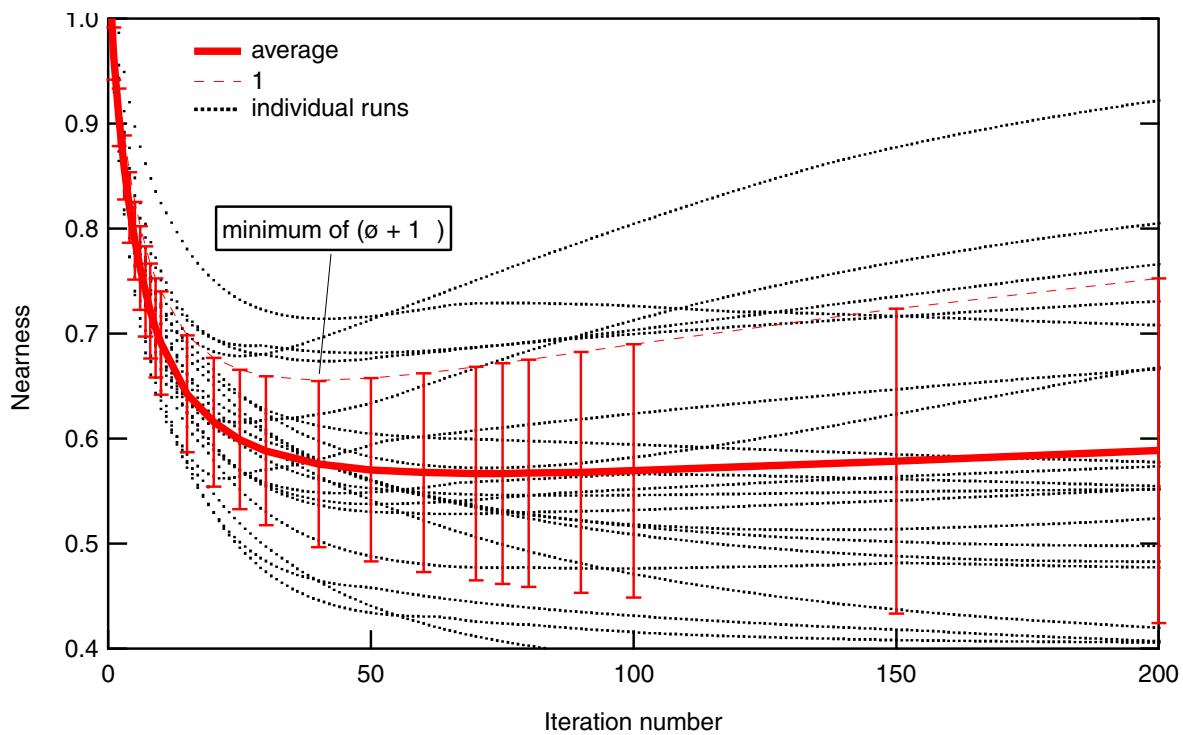

Fig. 5. Minimization in respect to $(\phi+1 \sigma)$ of the nearness for one specific test field. The simulation of the measurement and the SIRT reconstruction are applied 1000 times to a single test field with normal distributed measurement errors $1 \sigma=1000 \mathrm{ppb} * \mathrm{~m}$ (around $10 \%$ relative error). After 40 iterations, the average nearness values still decrease slightly with the iteration number, whereas the scattering of the nearness values increases strongly. As a compromise between a small average $\phi$ and a small scattering of the nearness values, the iteration number is chosen that minimizes $(\phi+1 \sigma)$.

\subsubsection{Normalized maximum difference (NMD)}

The normalized maximum difference (NMD) is the normalized maximum absolute value of the reconstruction error.

$$
\begin{aligned}
& \mathrm{NMD}=\frac{1}{N} \max _{r \in F} \Delta c(\mathbf{r}) \\
& \text { with } \mathrm{N}=\frac{1}{2}\left(\max _{r \in F} c_{\text {real }}(\mathbf{r})-\min _{r \in F} c_{\text {real }}(\mathbf{r})\right)
\end{aligned}
$$

The normalization factor $\mathrm{N}$ is half of the range between the lowest and the highest concentration of the real field. The NMD is invariant to multiplication of the field by a scalar factor and to the addition of a constant function. It is a measure for the maximum error which occurs. 


\subsubsection{Normalized average difference (NAD)}

The normalized average difference (NAD) is the average of the reconstruction error in the area of interest - normalized by the average concentration of the real concentration field.

$\mathrm{NAD}=\frac{\langle\Delta c(\mathbf{r})\rangle}{\left\langle c_{\text {real }}(\mathbf{r})\right\rangle}=\frac{\int d r^{2} \Delta c(\mathbf{r})}{\int d r^{2} c_{\text {real }}(\mathbf{r})}$

The NAD is invariant to the multiplication of fields by a scalar factor. This quality criterion is a measure how well the average concentration in the area of interest (and also the total amount of the trace gas species) is reproduced by the reconstruction.

\section{Reconstruction optimization}

Many parameters are involved in a reconstruction process based on linear discretization and row acting methods:

- dicretization model type (box/bilinear)

- discretization grid size

- first guess of the concentration field

- iteration number of the RAM

All these parameters implicitly add some sort of a priori information. Due to the limited amount of measurement data, the parameters have to be chosen very carefully. Depending on the choice of the discretization model, for example, special kinds of concentration fields are favored.

The optimal set of reconstruction parameters is derived from another kind of a priori information: the assumed probability distribution of the measurement error $\Delta \boldsymbol{d}_{\text {meas }}$ and the assumed distribution of the random function real concentration field $c_{\text {real }}(\mathbf{r})$. The parameters will be optimized so that the real field will be reconstructed best in average. "Reconstructed best" means, that certain reconstruction quality criteria, e.g. the nearness, become minimal. The choice of quality criteria described in the previous section depends on the further use of the reconstructed trace gas map.

\subsection{Generation of the set of test plumes}

The probability distribution of the random function "real concentration field" $c_{\text {real }}(\mathbf{r})$ is realized by a set of test concentration fields $\left(c_{k}(\mathbf{r})\right)_{k \in N}$. This set is used for optimizing the reconstruction parameters and estimating the error. The aim is to generate random fields in physically reasonable boundaries. It is a compromise between covering all cases and not being too general without need. For this study one hundred test fields were generated by randomly modifying the CTM BAB II concentration field (see Fig. 4).

The following modifications which are a coarse representation of physical processes where chosen:
- log-normal distributed random squeeze in intensity (different source strength).

- log-normal distributed random squeeze in position space (different meteorological situations).

- normal distributed random shift in position (different meteorological situations and chemistry) .

- addition of a small scale random field (local fluctuations and emissions from other sources).

\subsection{Parameter optimization procedure}

To find an optimum set of parameters the following numerical experiment was used:

FOR ALL sets of parameters DO \{

FOR ALL test fields $c_{k}$ DO

\{

simulate measurement by forward modeling the data from the continuous field;

FOR $n$ random meas. errors DO

\{

add measurement error to data;

reconstruct state vector $\mathrm{s}$ and related concentration

field $\mathrm{c}(\mathrm{s}, \mathrm{r})$;

evaluate reconstruction quality criteria;

\}

evaluate $\phi+1 \sigma$

\}

evaluate $\langle\phi+1 \sigma\rangle$;

\}

choose set of parameters minimizing $\langle\phi+1 \sigma\rangle$;

As we will see from the numerical experiments, the optimum parameters depend on the size of the measurement errors. For illustrating the optimization procedure we use quality criterion "nearness" (Sect. 4.3.1.).

\section{LOOP A:}

First the algorithm for a fixed test field with random measurement error (inner loop) is studied: Due to the simulated noise on the measurements, the simulated data scatter. Then also the nearness of the reconstructed fields scatter around the average nearness value. In Fig. 5 the nearness is plotted against the iteration number using one fixed test field and different realizations of the normal distributed measurement error. As can be seen in Fig. 5 the average nearness still decreases slightly with increasing iteration number, when the scattering of the nearness values increases strongly. Striving for a compromise between low average nearness and low scattering of the nearness values, we store the average $\phi$ plus one standard deviation $\sigma$ of the nearness values. $(\phi+1 \sigma)$. If we optimized the iteration number with 


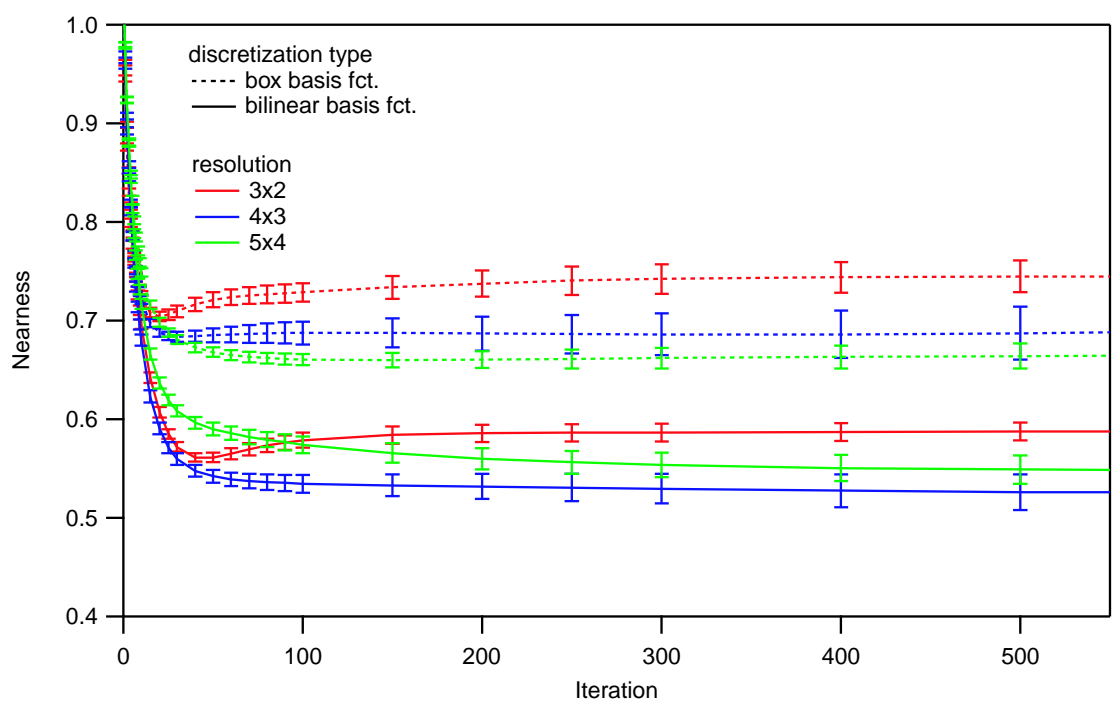

(a)

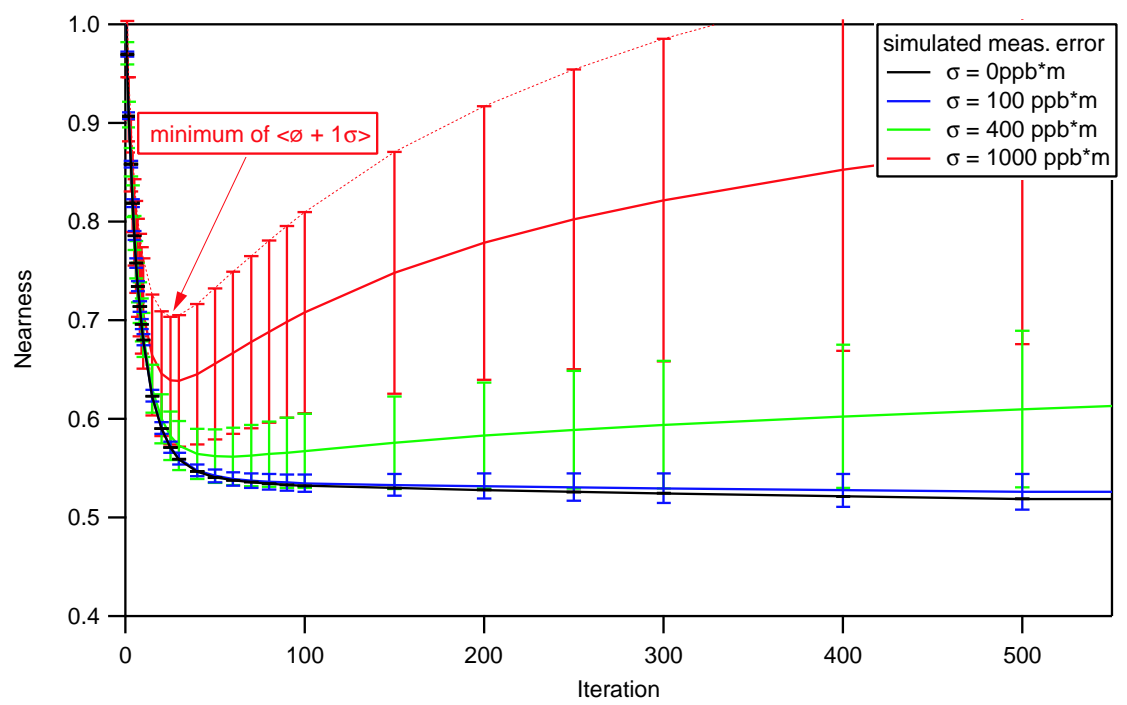

(b)

Fig. 6. Parameter optimization. The simulation of a measurement and the SIRT reconstruction are applied on 100 test-fields. The average nearness and its standard deviation averaged over all test-fields are plotted against the iteration number. The standard deviation shows the effect of the propagated measurement error on the nearness. In (a) different state models are compared for fix measurement error $\sigma=100 \mathrm{ppb} * \mathrm{~m}$. For all resolutions the bilinear discretization model is superior to the box model. The $4 \times 3$ grid leads to better results than the $3 \times 2 / 5 \times 4$ grids which correspond to strongly over/under determined problems. (b) For the fixed $4 \times 3$ bilinear state model the measurement errors are varied from $0-1000 \mathrm{ppb} * \mathrm{~m}$ (around $0-10 \%$ relative error). The optimum iteration number is determined by minimizing $\langle\phi+1 \sigma\rangle$. It is highly dependant on the measurement error and decreases when the simulated measurement error increases.

only one test field the optimum iteration number would be the one which minimizes $(\phi+1 \sigma)$. In Fig. 5, for example 40 iterations are optimal.

\section{LOOP B:}

Averaging over the set of test fields is done by storing the average of $(\phi+1 \sigma)$ over all fields: $\langle\phi+1 \sigma\rangle$.

\section{LOOP C:}

Finally, the optimum set of parameters is the one which minimizes $\langle\phi+1 \sigma\rangle$. 
Table 1. Results of the procedure for comparing reconstruction methods and inversion parameters. The optimization procedure was applied to the motorway setup used during the BAB II campaign. For different discretization models and inversion techniques the optimum iteration number $\mathrm{i}$ is given with respect to the three quality criteria nearness, normalized average difference (NAD), and normalized maximum difference (NMD). We took the optimum iteration number i for a minimum average plus one standard deviation of the quality criteria, thus making a compromise between the value of a quality criteria and its scattering of a quality criteria. The upper part of the table compares different discretization models for the fixed SIRT algorithm. The lower part compares different inversion techniques and their ideal iteration number depended on the assumed measurement error. In this part of the table the discretization model is fixed to be " $4 \times 3$ bilinear".

\begin{tabular}{|c|c|c|c|c|c|c|c|c|c|c|c|c|}
\hline \multirow{2}{*}{$\begin{array}{l}\text { Meas. Err. } \\
\sigma_{\text {meas }}\end{array}$} & \multirow{2}{*}{ RAM. } & \multirow{2}{*}{$\begin{array}{l}\text { Disc. } \\
\text { mod. }\end{array}$} & \multirow{2}{*}{$\begin{array}{l}\text { Grid- } \\
\text { size }\end{array}$} & \multicolumn{3}{|c|}{ Nearness } & \multicolumn{3}{|c|}{ NAD } & \multicolumn{3}{|c|}{ NMD } \\
\hline & & & & $<\Phi+1 \sigma>_{\min }$ & $1 \sigma$ & $\mathrm{i}$ & $<\Phi+1 \sigma>_{\min }$ & $1 \sigma$ & $\mathrm{i}$ & $<\Phi+1 \sigma>_{\min }$ & $1 \sigma$ & $\mathrm{i}$ \\
\hline 100 & SIRT & box & $3 \times 2$ & 0.71 & 4.84E-03 & 20 & 0.0103 & 0.0064 & 40 & 0.64 & $7.66 \mathrm{E}-03$ & 150 \\
\hline 100 & SIRT & Bil & $3 \times 2$ & 0.57 & $4.30 \mathrm{E}-03$ & 40 & 0.0114 & 0.0063 & 40 & 0.53 & $7.86 \mathrm{E}-03$ & 2000 \\
\hline 100 & SIRT & box & $4 \times 3$ & 0.69 & $4.85 \mathrm{E}-03$ & 30 & 0.0206 & 0.0157 & 1000 & 0.60 & 8.04E-03 & 40 \\
\hline 100 & SIRT & Bil & $4 \times 3$ & 0.53 & 8.83E-03 & 100 & 0.0130 & 0.0091 & 400 & 0.47 & $1.36 \mathrm{E}-02$ & 500 \\
\hline \multirow[t]{2}{*}{100} & SIRT & box & $5 \times 4$ & 0.67 & $6.02 \mathrm{E}-03$ & 100 & 0.0309 & 0.0073 & 2000 & 0.58 & $1.13 \mathrm{E}-02$ & 200 \\
\hline & SIRT & Bil & $5 \times 4$ & 0.56 & $1.43 \mathrm{E}-02$ & 500 & 0.0218 & 0.0076 & 250 & 0.45 & $1.86 \mathrm{E}-02$ & 1000 \\
\hline 100 & ART & Bil & $4 \times 3$ & 0.56 & $1.58 \mathrm{E}-02$ & 10 & 0.0174 & 0.0136 & 30 & 0.49 & $1.24 \mathrm{E}-02$ & 4 \\
\hline 1000 & ART & Bil & $4 \times 3$ & 0.84 & 0.1102404 & 1 & 0.1029 & 0.0778 & 6 & 0.60 & $8.51 \mathrm{E}-02$ & 2 \\
\hline 100 & SART & Bil & $4 \times 3$ & 0.54 & $1.51 \mathrm{E}-02$ & 40 & 0.0093 & 0.0063 & 6 & 0.47 & $1.54 \mathrm{E}-02$ & 100 \\
\hline 1000 & SART & Bil & $4 \times 3$ & 0.75 & $6.26 \mathrm{E}-02$ & 4 & 0.0626 & 0.0614 & 6 & 0.62 & 0.070997 & 10 \\
\hline 100 & SIRT & Bil & $4 \times 3$ & 0.53 & 8.83E-03 & 100 & 0.0130 & 0.0091 & 400 & 0.47 & $1.36 \mathrm{E}-02$ & 500 \\
\hline 1000 & SIRT & Bil & $4 \times 3$ & 0.70 & $6.47 \mathrm{E}-02$ & 25 & 0.0716 & 0.0597 & 80 & 0.58 & 7.78E-02 & 50 \\
\hline
\end{tabular}

\subsection{Parameter optimization for the motorway setup}

The numerical optimization procedure was applied to the measurement setup used during the BAB II campaign (Fig. 1a). As first guess a constant field with the average concentration of the measured light-paths was used which proved to be suitable in preliminary examinations. Some optimization results for different quality criteria are presented in Table 1. For different discretization models and types of row acting methods the optimum iteration number is given. As we were interested in the overall shape of the plume, we decided to employ the quality criterion "nearness" for the rest of this study. The optimization is graphically illustrated in Fig. 6. In Fig. 6a the Nearness is plotted against the iteration number for different state models using the SIRTalgorithm. The bilinear discretization model is superior to the box model because of the smaller discretization error. The combination of a simultaneous row acting method and a bilinear discretization model on a $4 \times 3$ grid seems to be best. In Fig. $6 \mathrm{~b}$ the dependence of the optimal iteration number on the measurement error is demonstrated. If the measurement error increases, the ideal iteration number decreases. The optimum iteration number in our case is about 100 for a measurement error of $100-400 \mathrm{ppb} * \mathrm{~m}$ which is about $2 \%$ relative error and corresponds to the measurement error during the motorway campaign. In spite of the optimization, all nearness values are relatively high, as the source region above the carriageway where large concentration gradients occur is poorly covered by the light paths. This problem can be seen more clearly in the 2-D error fields and reconstructed concentration fields of the next sections.

\subsection{General observations}

Applying the optimization procedure to different measurement setups (motorway campaign and other atmospheric setups) the following general observations were made:

- As discretization model the bilinear interpolation model is superior to the box model.

- Comparing the reconstruction quality of different row acting methods, the SIRT-like inversion method was best in the presence of noise on the data, closely followed by SART. The ART-like method yielded distinctly worse results.

- The convergence is much faster for SART than for SIRT, but in our case this is not of interest because both algorithms are very fast for our problem size.

- If the grid size is optimal the inverse problem is only just or just not anymore well determined. In other words the resolution matrix $\mathbf{R}=\mathbf{F}^{\dagger} \mathbf{F}$ which should be close to unity is nearly of full rank. If the grid size is smaller the discretization error gets large and the result gets worse.

\section{Reconstruction results}

The meteorological situation of the day of the study was a weak anticyclonal circulation, clear sky conditions and light to moderate winds. After a nightly inversion due to radiative cooling, a shallow convective layer developed in the diurnal 
Table 2. Optimized reconstruction parameters for the motorway campaign.

\begin{tabular}{ll}
\hline Discretization model & $4 \times 3$ bilinear \\
RAM type & simult. iterative projection method \\
First guess & constant average \\
Iteration Number & 100 \\
\hline
\end{tabular}

cycle which reached $1500 \mathrm{~m}-2000 \mathrm{~m}$ altitude. On synaptic scales the pressure differences were small; therefore the advective processes were not dominating.

For reducing the stepping error the $\mathrm{NO}_{2}$ data from the BAB II-campaign had to be averaged over several hours. Because of the linearity of the reconstruction process (in the inversion process each projection of the row acting method is linear and the state model is linear), this averaging of the data equals averaging the 2-D-concentration fields. Therefore the reconstructed fields are time-average concentration fields over the selected periods. The problem of temporal changes of the concentration field during one stepping cycle which result in stepping errors is discussed in the next section.

Searching time periods in which the wind direction is approximately perpendicular to the motorway and taking into account the measurement periods of the IMK-Karlsruhe we chose three time periods.

\section{Period 110 May 2002 06:00-10 May 2002 13:00 CET}

Period 210 May 2002 13:00-10 May 2002 19:00 CET

Period 3 10 May 2002 20:00-11 May 2002 01:00 CET

Figure 7 shows the reconstructed $\mathrm{NO}_{2}$ concentration fields for the three time periods. We used the reconstruction parameters optimized for this setup, as listed in Table 2. The shape of the exhaust gas plume depends on the strength of the source (vehicle flux), the chemistry, the wind-speed, the wind-direction and the atmospheric stratification. During situations with stable stratification the exhaust gas concentrations increase strongly - especially if the wind speed orthogonal to the motorway is low. Because the main sources of $\mathrm{NO}_{2}$ at the motorway are chemical reactions and not direct emission, the concentration maximum is on the downwind side of the motorway. The IMK Karlsruhe performed measurements of the vertical wind and temperature profiles during the campaign (M. Kohler, personal communication). The wind vectors measured at two different altitudes are shown in Fig. 8. The shape of the reconstructed plumes agrees with expectations based on vehicle fluxes (B. Vogel, personal communication) and meteorological conditions. Time period 1 (Fig. 7a) includes

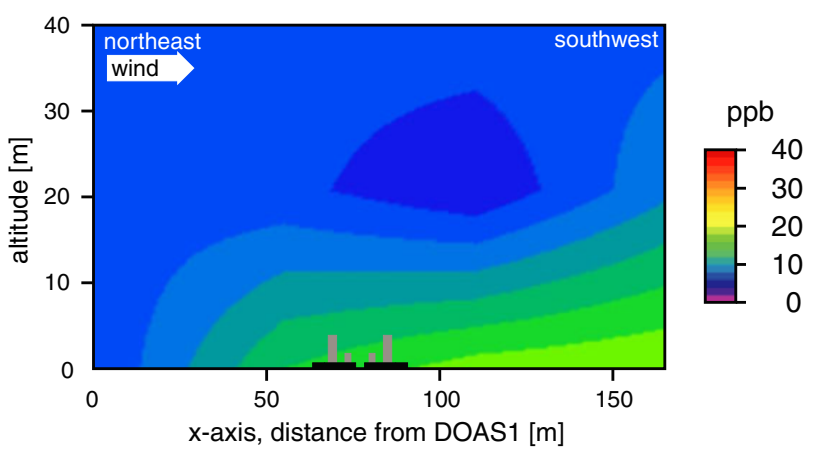

(a) Period1 (06.00-13.00h)

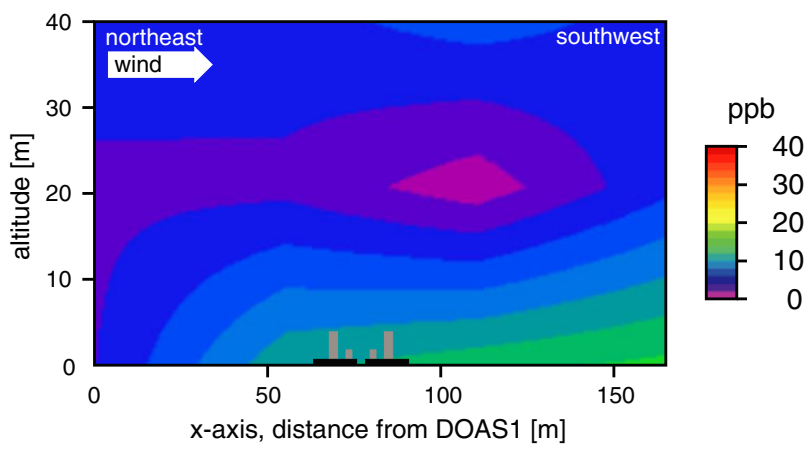

(b) Period2 (13.00-19.00h)

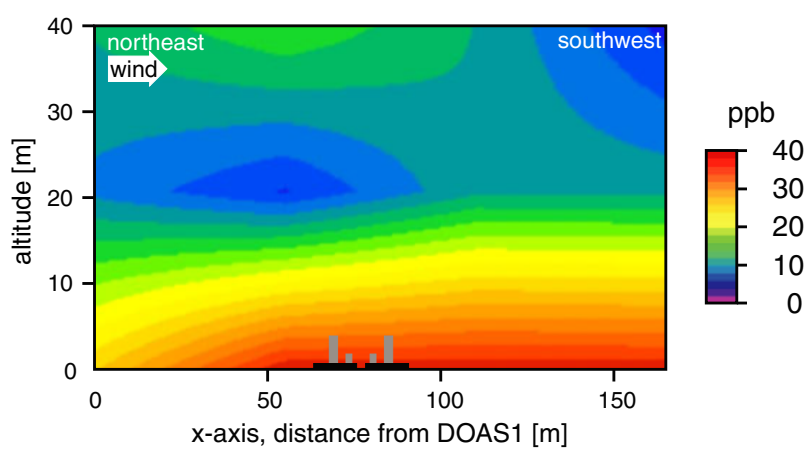

(c) Period3 (20.00-01.00h)

Fig. 7. Reconstruction results for the BAB II campaign. The reconstruction was performed using the simultaneous row acting inversion method and a $4 \times 3$ bilinear interpolation model (SIRT-like). The reconstructed concentration fields are in good agreement with the meteorological situation. During day time the exhaust gases were driven away from the carriageway by a soft breeze. Due to the morning rush hour the plume is stronger in period 1. At nighttime the wind-speed at ground-level was almost zero and the temperature gradient showed an inversion situation which leads to high concentrations (also in the background air).

the morning rushhour around 6 a.m. CET which coincides with a stable stratification. Around 11 a.m. a convective layer develops and dominates time period 2 (Fig. 7b). 


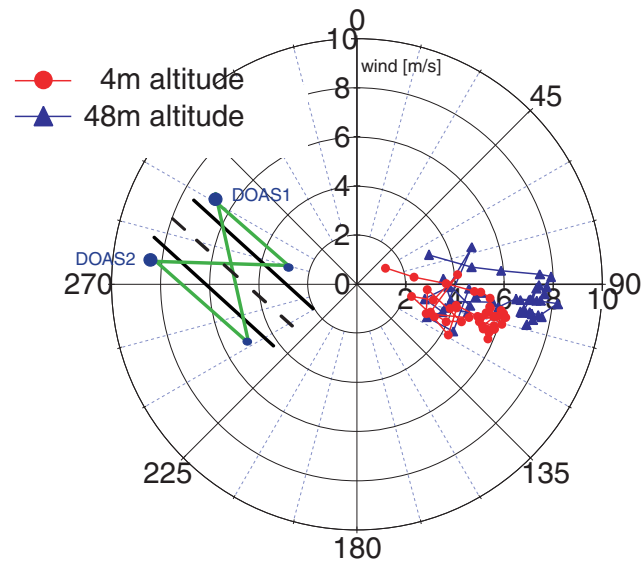

Period 1 (06:00-13:00h)

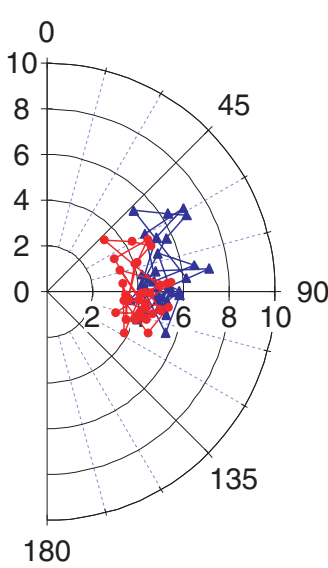

Period 2 (13:00-19:00h)

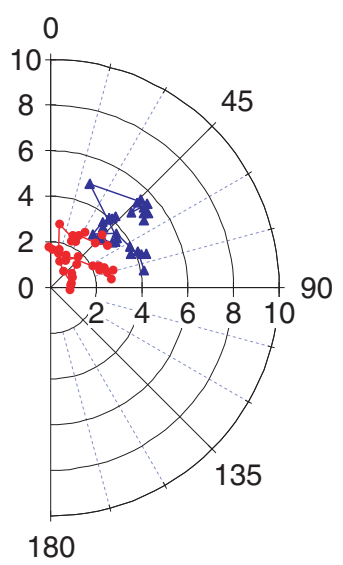

Period 3 (20:00-01:00h)

Fig. 8. Wind direction BAB II. The wind direction at 4 and $50 \mathrm{~m}$ altitude above the motorway is plotted for the three time periods. The wind component vertical to the motorway is the smallest during time period 3 .

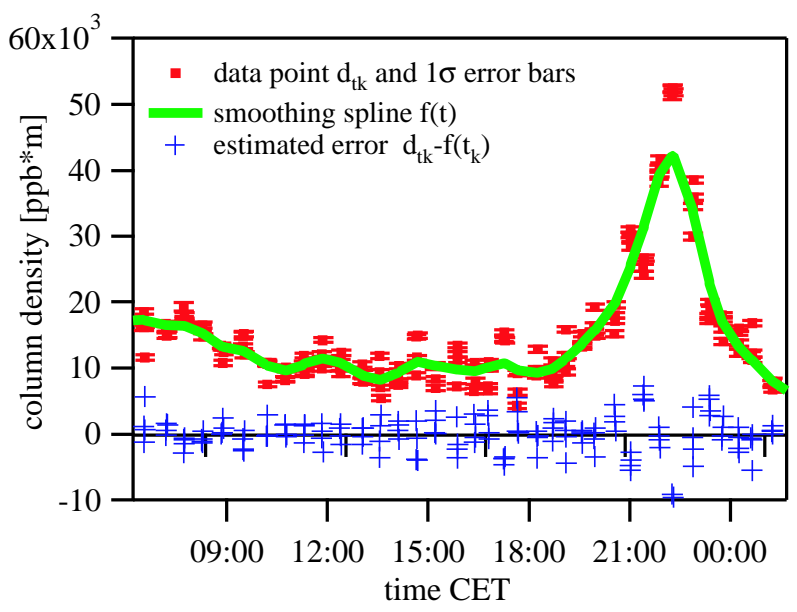

Fig. 9. Estimation of the stepping error. The scattering of the measurement data is not primarily due to insufficient measurement devices, but to real atmospheric and vehicle emission fluctuations during the stepping process. The measured $\mathrm{NO}_{2}$ slant column densities (red) for one lightpath are plotted against the time. The stepping error of an individual data point (blue) is estimated to be its distance from an interpolating smoothing spline (green).

Consequently, the $\mathrm{NO}_{2}$ concentrations are distinctly lower. In both periods ( 1 and 2 ) the plume is driven away to the right of the carriageway by a soft breeze.

During time period 3 (Fig. 7c) the ground wind speed gets very slow. Together with a stable stratification and thus a very low boundary layer this leads to a strong accumulation of $\mathrm{NO}_{2}$. In this time period, at around $40 \mathrm{~m}$ altitude, concentrations 5-10 ppb higher than the background concentration were reconstructed. Because no lightpath is crossing this upper area (see Fig. 4a) this feature is likely to be an artifact of the reconstruction. By considering the reconstruction error which is quantified in the following section the lack of significance of this feature is confirmed. Also the exact position of the plume maximum cannot be determined well with this measurement setup as one can see in the error maps Fig. 10a, b, Fig. 11a and Fig. 12a, c. which are described in Sect. 7. For instance it is possible that the real maximum in period 1 and 2 is located closer to the motorway.

It is interesting to note that the plume shapes and concentration values correspond well to the simulated $\mathrm{NO}_{2}$ profiles of Bäumer (2003). In his simulations for the same motorway situation, emission data and wind data from a former campaign were used. The results of his simulations for 13:00 CET (unstable stratification) show similar features (plume height, absolute concentration) as our reconstructions from period 1/2, the ones for 21:00 CET (stable stratification) as period 3 .

\section{Estimated reconstruction error}

After sketching the determination of the measurement error on the data, our procedure for estimating the measurement error and the discretization + inversion error are described. Resulting 1-D cross sections and 2-D error maps are presented for the real data and the CTM BAB II plume, also with an improved motorway measurement setup.

\subsection{Stepping error}

In our case, the stepping error is the dominating part of the measurement error. It arises when the stepping technique is used, i.e. different light paths are not measured simultaneously but successively. Then the data from different light paths refer to different times. Hence the measured data has to be taken for an estimate of the average value along the 


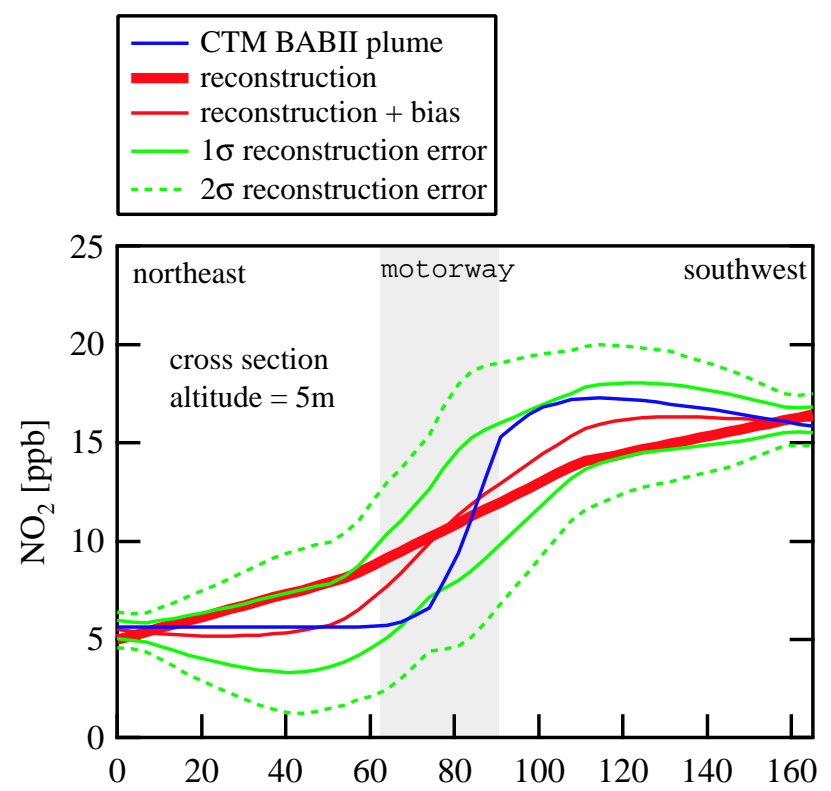

a) $\mathrm{NO}_{2} \mathrm{CTM}$ BABII plume with BABII setup
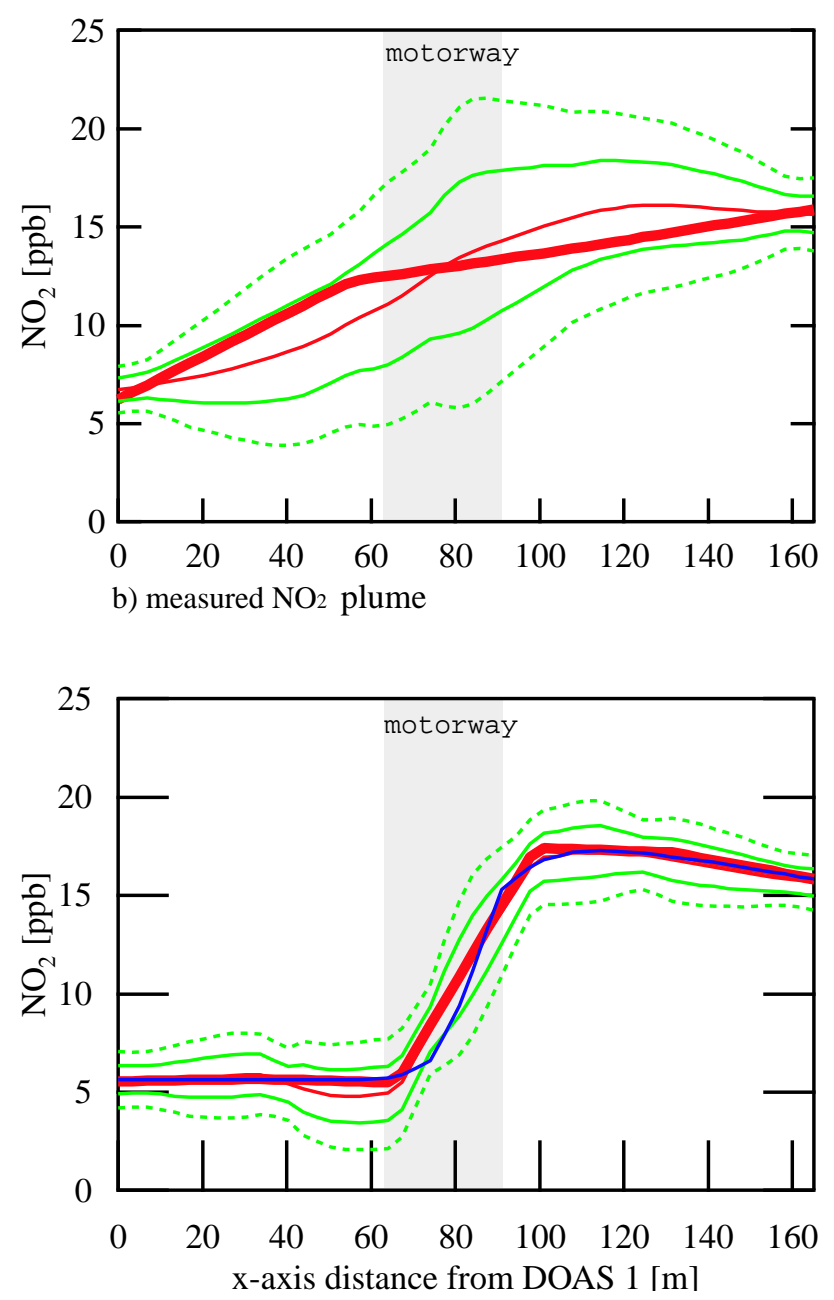

c) $\mathrm{NO}_{2}$ CTM BABII plume with enhanced setup

Fig. 10. 1-D cross sections of concentration fields and error estimations at $5 \mathrm{~m}$ altitude. (a) Measurements are simulated on the CTM BABII plume using the BABII setup. The inversion is performed on the simulated data. The reconstructed plume (red) differs from the original (blue), but remains everywhere within estimated $1 \sigma$ error limits (green). In the middle area $1 \sigma$ is around 5 ppb which allows only qualitative statements about the shape of the plume. (b) The inversion is performed on the measured data from period 1. Again only qualitative statements about the shape of the plume are possible. The discretization and inversion part of the error is the same as in (a) but the measurement error part differs from (a) because the estimated measurement error from the measurement data is used (Sect. 7.1). (c) Measurements are simulated on the CTM BABII plume using the enhanced setup. (Fig. 1b, Sect. 7.6). Again the original and reconstructed concentrations are shown. Here the shape of the plume can be grasped with much better accuracy. The position of the concentration peak can be determined well. 


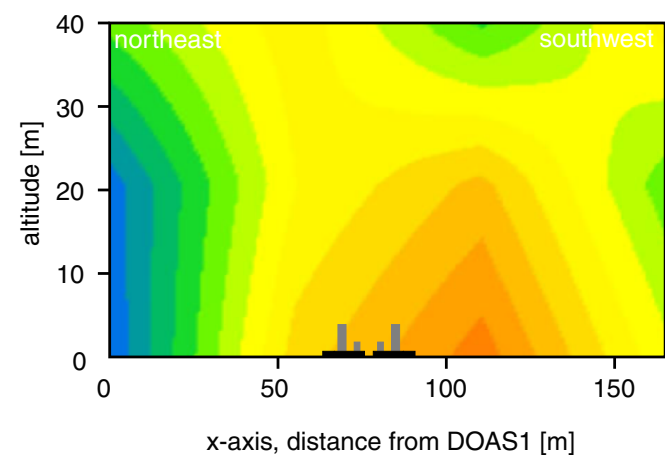

(a) std. dev. map $\sigma(\Delta$ cmeas $)$, original setup

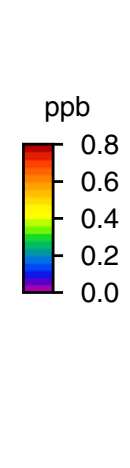

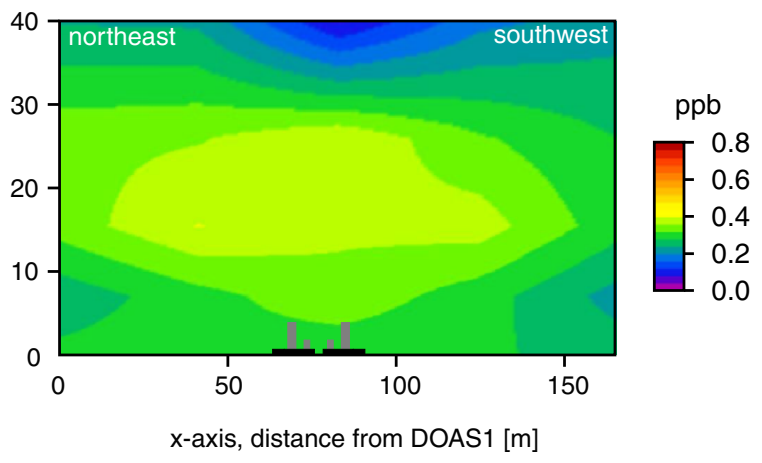

(b) std. dev. map $\sigma(\Delta$ cmeas), enhanced setup

Fig. 11. Measurement error. Subfigure (a) shows the propagated measurement error for the BAB II setup at time period 1. The error is the largest close to the carriageway. This is due to the poor coverage of this region with light beams. The shifting to the right results from higher fluctuations of the concentrations and thus a higher absolute measurement error on the right hand side of the motorway in this wind situation. Subfigure (b) shows the measurement error map for the enhanced motorway setup. The error on the data was assumed to be the same for all light paths and of average size of the errors in subfigure (a). One must be careful in comparing (a) and (b) as the choice of taking the same error for all light paths in (b) already results in a smoother and more symmetric field. Independent of the spatial distribution the average resulting error is smaller than in (a) because more light paths are in the area.

light paths. If the observed concentration field shows temporal fluctuations, e.g. if vehicles are passing the measurement site, then the measurement data scatters around the average. This scattering produces the stepping error. The stepping error can be reduced by averaging the data over a time period, smoothing or interpolating the data. We averaged the data to get data values $\bar{d}$. The stepping error was estimated in the following way: The measurement data as a function of time is interpolated with a smoothing spline (IGOR Pro, Wave Metrics, Inc., with reference to Reinsch, 1967) where for each measurement point its measurement error derived from the DOAS analysis is taken into account. The smoothness parameter of the interpolation method was chosen by eye. The distance of the measurement data from the interpolating spline is taken as the stepping error of a single data point. From this the standard error of the mean is calculated:

$\bar{d}=\frac{1}{K} \sum_{k=1}^{K} d_{t k} ; \quad \Delta \bar{d}=\sqrt{\frac{\sum_{k=1}^{K}\left(d_{t_{k}}-f\left(t_{k}\right)\right)^{2}}{(K-1) K}}$,

where $d_{t k}$ are the data points at time $t_{k}, f(t)$ is the interpolating spline curve and $K$ is the sample size of the period to be averaged. The stepping error was estimated separately for all light paths. In Fig. 9 this estimation is shown for one lightpath. The measured slant column densities, the interpolated spline and the resulting estimated errors for each single point are plotted. The resulting relative error for the lightpaths was about $2 \%$ of the average column densities.

\subsection{Propagated measurement error}

We assume that the measurement error on the data, which in our case is the stepping error described in the previous section, is independent and Gaussian distributed. Then we can calculate the measurement error in the reconstruction $\Delta c_{\text {meas }}(\mathbf{r})$ from the covariance of the measurement error on the data covd $\boldsymbol{d}_{\text {meas }}$ with Eq. (18) from Sect. 4.2. The diagonal elements of $\operatorname{cov} \boldsymbol{d}_{\text {meas }}$ correspond to the stepping errors. From $\operatorname{covc}_{\text {meas }}$ a standard deviation map is obtained which gives an impression about the insecurity of the reconstruction at different points due to the measurement error on the data.

\subsection{Discretization and inversion error}

As described in Sect. 4.2. we estimate the discretization + inversion error by a numerical experiment which corresponds to Eq. (17). We simulate the measurement and the reconstruction on a set of test fields $\left(c_{k}(\mathbf{r})\right)_{k \in N}$ which are described in Sect. 5.1. They are based on the CTM-BABII plume (Bäumer pers. comm.) which is shown in Fig. 4a. The difference between the resulting concentration fields and the test fields, $\Delta c_{k}(\mathbf{r})$, is evaluated statistically to get the error fields:

$$
\begin{aligned}
\left\langle\Delta c_{d i}\right\rangle(\mathbf{r}) & =\frac{1}{k} \sum_{k=1}^{K} \Delta c_{k}(\mathbf{r}) \\
\sigma\left(\Delta c_{d i}\right)(r) & =\sqrt{\frac{1}{K} \sum_{k=1}^{K}\left(\Delta c_{k}(\mathbf{r})^{2}-\left\langle\Delta c_{d i}\right\rangle(\mathbf{r})\right)}
\end{aligned}
$$

The distribution of the discretization and inversion error $\Delta c_{d i}(\mathbf{r})$ is not symmetric around zero. Therefore we always show the average map $\left\langle\Delta c_{d i}\right\rangle(\mathbf{r})$ and the standard deviation map $\sigma\left(\Delta c_{d i}\right)(\mathbf{r})$ to get an impression about the quality of the reconstruction at each point. 


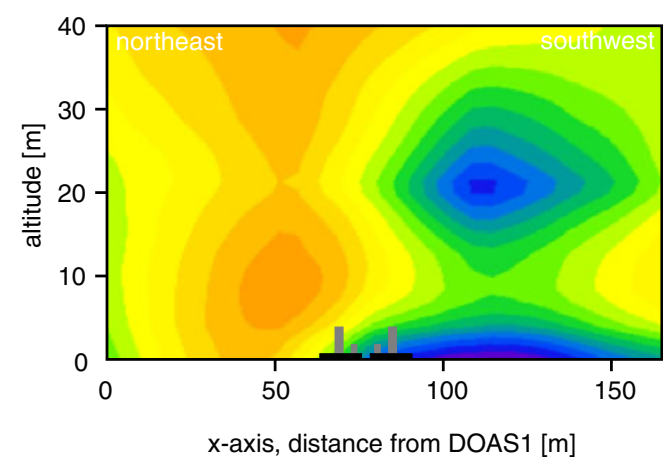

(a) average map $<C_{d i}$, original setup

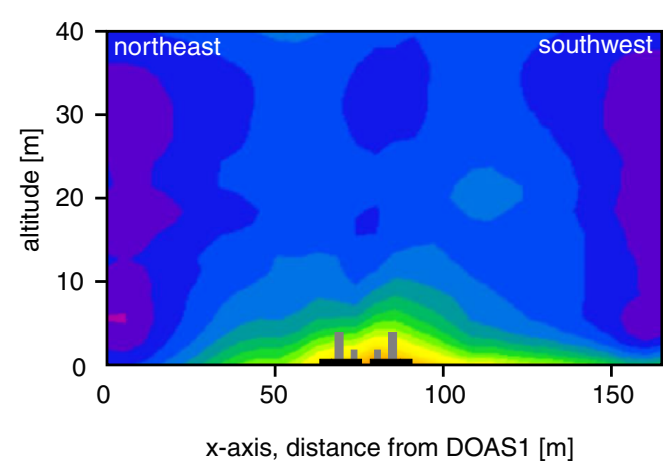

(c) std. dev. map $\sigma\left(\Delta c_{d i}\right)$, original setup

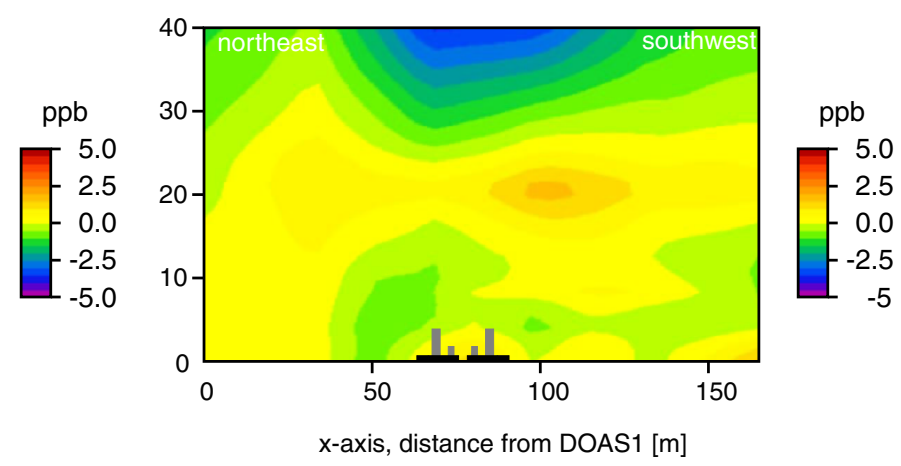

(b) average map $<C_{d i}$, enhanced setup

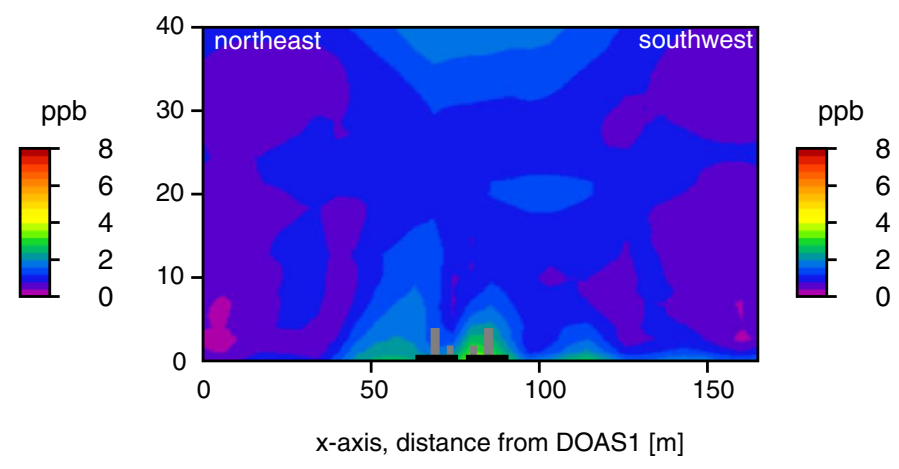

(d) std. dev. map $\sigma\left(\Delta c_{d i}\right)$, enhanced setup

Fig. 12. Discretization and inversion error. The combination of discretization and inversion error was determined by a numeric experiment with a set of test plumes based on the CTM BAB II plume for the north-east wind situation. This error is not symmetric around zero, therefore we plot the average (a, b) and the std. dev. (c, d) of the error distribution. Subfigure (a) and (c) refer to the original setup. (a) The concentration is underestimated where high concentration values are likely, but overestimated in the other regions: The plume is smoothed, because the discretization model is too low resolved and cannot represent the steep concentration gradient which is likely to occur over the motorway. (c) The standard deviation is the highest above the road, because the coverage with light beams is insufficient, and smallest on the sides of the road where a lot of light beams are available. For the enhanced setup the average deviation (b) is generally low (ignoring the area high above the road, which is not of scientific interest). The standard deviation (d) exceeds 2 ppb only in the lowest $2 \mathrm{~m}$ above the carriageway. Because of the traffic no light beams could be located here.

\subsection{1-D cross-sections of concentration fields}

Before discussing the 2-D-error maps we present 1-D cross sections of these maps. The plots in Fig. 10 show horizontal $\mathrm{NO}_{2}$ concentration cross-sections perpendicular to the carriageway, $5 \mathrm{~m}$ above ground. Additionally the $1 \sigma$ and $2 \sigma$ reconstruction error limits are plotted. They are derived by adding the average and the standard deviation of the estimated error $\Delta c(\mathbf{r})$ to the reconstruction result. It was tested in a numerical experiment that in the given situation adding up the standard deviations of $\Delta c_{\text {meas }}(\mathbf{r})$ and $\Delta c_{d i}(\mathbf{r})$ quadratically is a good approximation for folding the distributions.

For Fig. 10a a theoretical measurement was simulated on the CTM BAB II plume and the concentration distribution reconstructed from the column densities. The uncertainty of the concentration in the middle area and therefore the differ- ence between the original and its reconstruction is high. This is due to the missing coverage of lightbeams directly above the carriageway on the one hand and the high concentration gradients near the source on the other hand. The a priori information which is implicitly used in the underdetermined region smoothes the result. Another reason for the difference is that the $4 \times 3$ bilinear interpolation model, which can be recognized by the four sharp bends of the reconstructed curve, cannot grasp the CTM BAB II plume.

In Fig. $10 \mathrm{~b}$ we show the cross section of the $\mathrm{NO}_{2}$ concentration at time period 1 (taken out of the 2-D field Fig. 7a). The estimated reconstruction error consists of the same discretization and inversion error part $\Delta c_{d i}(\mathbf{r})$ as in Fig. 10a and a different propagated measurement error part derived from the measured data. In the center area the $1 \sigma$ uncertainty is around $5 \mathrm{ppb}$. Near the borders, where we have good light beam coverage, it diminishes to $1 \mathrm{ppb}$. The 
reconstruction quality is good enough to determine qualitatively a "plume type" like "evening inversion plume" or "strong wind plume". For quantitative statements this measurement setup works only in the areas of the vertical profiles on both sides. One might have the idea, that $\left(c_{\text {rec }}(\mathbf{r})+\langle\Delta c(\mathbf{r})\rangle\right)$ (the fine red curve) is a better estimation for the real field than $c_{r e c}(\mathbf{r})$. This was true, if the a priori information represented by the set of model plumes was exactly right. As we don't know the reliability of this information and don't want to force the reconstruction result towards the CTM BAB II plume which was used to generate the test fields, we don't pursue this idea.

\subsection{2-D error maps}

In the error maps Figs. 11 and 12, respectively, the propagated measurement error field $\Delta c_{\text {meas }}(\mathbf{r})$, and the discretization and inversion error field $\Delta c_{d i}(\mathbf{r})$ are shown separately. With these maps, the uncertainty can be estimated for each point of the field by adding the two standard deviation error fields statistically and taking the average map $\left\langle\Delta c_{d i}(\mathbf{r})>\right.$ into account.

Additionally to the effects explained in Sect. 7.4 the reconstruction is less accurate directly above the motorway, and more accurate at higher altitude than at five meter (Fig. 10). The standard deviation due to the measurement error (see Fig. 11) - shown for time period 1 - is relatively small compared to the other errors. The average maps of $\Delta c_{d i}(\mathbf{r})$ show that generally the concentrations are underestimated at the location of the plumes, and overestimated in the other regions. This "watering down" is caused by the discretization error (the state model is not fine enough to describe the plume position accurately) and due to a general smoothing of SIRT at low iteration numbers in underdetermined regions. In our setup the inversion + discretization error due to the few measurements is of the order of ten times higher than the propagated measurement error.

\subsection{Reduced errors with an improved measurement setup}

The uncertainties of the reconstructions require an improved measurement setup which is able to quantify the plume shape and concentration accurately over the entire field and which can be used for possible future campaigns.

In numerical experiments we added two more DOAS telescopes between the existing telescopes and one additional retro reflector on a bridge at $5 \mathrm{~m}$ altitude above the motorway (see Fig. 1b). Instead of conventional telescopes, four multibeam telescopes (a new type of telescope which is able to measure several light paths simultaneously) are used for measuring six light paths simultaneously. These telescopes measure four light paths which are stepping between two retroreflectors, respectively, one fix light path to the bridge retroreflector (passing the most fluctuating part) and one light path for intercalibration purposes. The averaging time, and hence the time resolution are therefore reduced by a factor of five preserving the actual stepping errors size. The 36 light paths enable us to choose a higher resolved state model. A $6 \times 4$ bilinear interpolation model yielded the best reconstruction results with SIRT in the optimization process.

For the error studies the data errors were assumed to be identical for all light paths and of average size of the errors of the campaign time period 1. In Fig. 10c the 1-D cross section of the CTM BAB II plume and its reconstruction are shown for the new measurement setup. The difference between the original plume and the reconstruction is smaller than $1 \mathrm{ppb}$, and the $1 \sigma$ limits are lower than $2 \mathrm{ppb}$ (even in the problematic central area).

In Fig. 11b and Fig. 12b, c the two types of errors are shown separately on 2-D maps. The asymmetry in the measurement error map is due to the slightly asymmetric position of the retro-reflector towers. Only the lowest two meters above the motorway cannot be reconstructed very well as DOAS- light beams cannot be used at this altitude because of the cars. Also the top middle region can't be well determined by this setup but this area is not so important from the scientific point of view.

\section{Conclusions}

We have presented a procedure for comparing different reconstruction techniques and optimizing the reconstruction parameters for DOAS tomography measurements. The simulations of the measurement and the reconstruction were applied to a set of test plumes for different combinations of reconstruction techniques and parameters to find the optimal reconstruction algorithm and set of parameters. The set of test plumes represent all concentration fields which are physically reasonable according to our a priori information. For judging the reconstruction quality the quality criterion "nearness" was used in this study. For other purposes we suggested the criteria "normalized maximum difference" (NMD) and "normalized average difference" (NAD).

We used the procedure for comparing different row acting methods (ART-, SIRT-, and SART-like) and two types of discretization models (box, bilinear interpolation). The procedure can also be employed to optimize measurement setups. In the presence of noise on the data, an extension of the simultaneous iterative projection method SIRT with a bilinear discretization model yielded the best reconstruction results. The SART algorithm was slightly inferior and the ART-like inversion method, came off distinctly worse.

We applied the optimization, reconstruction and error estimation procedure to the data of the first tomographic DOASmeasurements carried out during a motorway emission campaign. For this setup with 16 light beams the extension of SIRT with a $4 \times 3$ bilinear interpolation model worked best. The optimum iteration number depends strongly on the measurement error. For the BAB II setup it is about 100 iterations 
for a relative average measurement error of $2 \%$. The measurement data had to be averaged over several hours because of the stepping error. The reconstructed $\mathrm{NO}_{2}$ fields for three time periods agreed well with the prevailing meteorological conditions. The estimated reconstruction error of $\sigma=5 \mathrm{ppb}$ (around 30\% relative error) above the carriageway is still quite large however, so that the exact location and the exact value of the concentration maximum can't be determined from our measurements. This can be seen quite clearly in the presented horizontal 1-D cross sections, on which the $1 \sigma$ error limits are marked.

For the error estimation we devided the reconstruction error into propagated measurement error on one side and discretization and inversion error on the other. This helps making suggestions to improve the measurements. If the propagated measurement error is too high, one should try to reduce the error on the data (e.g. by averaging the data over a longer period of time). If the discretization and inversion error is too high, one should rather modify the measurement setup. In our field-campaign setup the discretization and inversion error was one magnitude higher than the propagated inversion error.

As a consequence, an improved hypothetical motorway setup with 36 light beams was proposed. Assuming the same average measurement error as during the BAB II campaign, the concentration several meters above the carriageway can be reconstructed within 1-2 ppb. Using the new multibeam telescopes (a new type of DOAS-telescopes which can measure several light paths simultaneously) such a setup could be realized with a time resolution of 10 to $60 \mathrm{~min}$.

Summing up, the first DOAS tomography measurement and the applied reconstruction and error estimation procedures worked out successfully. Measurement and expectation seem to agree well within the given error limits. With the new measurement devices it is now possible to produce high quality 2-D trace gas maps by DOAS tomography.

Acknowledgements. We would like to thank the IMK Karlsruhe for the successful cooperation during the measurement campaign. Special thanks to D. Bäumer for putting the CTM BAB II plume at our disposal and M. Kohler and T. Kunz for their kind help in elucidating the meteorological situation. We'd also like to thank T. Wagner for the fruitful discussions and his help with writing this manuscript. This study was performed as part of the project "DOAS tomography: high quality 3-D measurements of tropospheric trace gases" funded by the German atmospheric research program AFO 2000 (Project 07 ATC-03, Young researchers fellowship program for research groups, AFO 2000-C).

Edited by: M. Ammann

\section{References}

Bäumer, D.: Transport und chemische Umwandlung von Luftschadstoffen im Nahbereich von Autobahnen - Numerische Simulationen, Ph.D. thesis, Fakultät für Physik, Universität Karlsruhe, 2003.

Censor, Y., Eggermont, P. P. G., and Gordon, D.: Strong underrelaxation in kaczmarz's method of inconsistent systems, Numerische Mathematik 41, 83-92, 1983.

Drescher, A. C., Gadgil, A. J., Price, P. N., and Nazaroff, W. W.: Novel approach for tomographic reconstruction of gas concentration distributions and simulated annealing, Atmos. Environ., 30, 929-940, 1996.

Drescher, A. C., Park, D. Y., Yost, M. G., Gadgil, A. J., Levine, S. P., and Nazaroff, W. W.: Stationary and time-dependent indoor tracer-gas concentration profiles measured by OP-FTIR remote sensing and SBFM-computed tomography, Atmos. Environ., 31, 727-740, 1997.

Fehmers, G. C., Kamp, L. P. J., and Sluijter, F. W.: An algorithm for quadratic optimization with one quadratic constraint and bounds on the variables, Inverse Problems, 14, 893, 1998.

Fiedler, F., Corsmeier, U., and Vogel, B.: Experimentelle Bestimmung der Emissionen des Kraftfahrzeugverkehrs auf Autobahnen und Vergleich mit berechneten Emissionen, Operationsplan, Selbstverlag des Instituts fur Meteorolgie und Klimaforschung, Forschungszentrum Karlsruhe/Universität Karlsruhe, 2001.

Fischer, M. L., Price, P. N., Thatcher, T. L., Schwalbe, C. A., Craig, M. J., Wood, E. E., Sextro, R. G., and Gadgil, A. J.: Rapid measurements and mapping of tracer gas concentrations in a large indoor space, Atmos. Environ., 35, 2837-2844, 2001.

Groetsch, C. W.: Inverse problems in the mathematical sciences, Vieweg, 1993.

Herman, G. T. and Rowland, S.: Three methods for reconstructing pictures from x-rays: A comparative study, Comput. Graph. Image Proc., 1, 151-178, 1973.

Herman, G. T., Lent, A., and Rowland, S.: ART: Mathematics and applications, a report on the mathematical foundations and on the applicability to real data of the algebraic reconstruction techniques, J. Theor. Biol., 42, 1-32, 1973.

Ingesson, L. C., Alpher, B., Chen, H., Edwards, A. W., Fehmers, G. C., Fuchs, J. C., Giannella, R., Grill, R. D., Lauro-Taroni, L., and Romanelli, M.: Soft x-ray tomography during ELMs and impurity injection set, Nuclear Fusion, 38, 1998.

Jiang, M. and Wang, G.: Convergence of the simultaneous algebraic reconstruction technique (sart), IEEE, 2001.

Kaczmarz, S.: Angenäherte Auflösung von Systemen linearer Gleichungen, Bull. Acad. Pol. Sci. Lett. A, 6-8A, 355-357, 1937.

Kak, A. and Slanley, M.: Principles of Computerized Tomographic Imaging, IEEE Press, 1988.

Knab, V.: Basic theory on DOAS-tomography - Reconstruction of 2-D trace gas distributions by discrete linear inversion techniques, Master's thesis, University of Heidelberg, 2003.

Kunz, C.: Charakterisierung eines Multi-Strahl Langpfad DOAS Systems zur Messung von Spurenstoffen in der Atmosphäre, Master's thesis, University of Heidelberg, 2003.

Perner, D., Ehhalt, D. H., Pätz, H. W., Platt, U., Röth, E. P., and Volz, A.: OH Radicals in the lower Troposphere, Geophys. Res. Lett., 3, 466-468, 1976.

Platt, U.: Dry deposition of $\mathrm{SO}_{2}$, J. Atmos. Environ, 12, 363-367, 1978. 
Platt, U.: Differential optical absorption spectroscopy (doas), in Monitoring by Spectroscopic Techniques, edited by Sigrist, M. W., John Wiley \& Sons, Inc., New York, 1994.

Price, P. N., Fischer, M. L., Gadgil, A. J., and Sextro, R. G.: An algorithm for real-time tomography of gas concentrations, using prior information about spatial derivatives, Atmos. Environ., 35, 2827-2835, 2001.

Pundt, I., Mettendorf, K.-U., Laepple, T., Knab, V., Xie, P., Lösch, J., v. Friedeburg, C., Platt, U., and Wagner, T.: Measurements of trace gas distributions by long-path DOAS-tomography: 2D mapping of $\mathrm{NO}_{2}$ during the motorway campaign BAB II, J. Atmos. Environ., accepted, 2004.

Reinsch, C. H.: Smoothing by spline functions, Numerische Mathematik, 1967.

Rodgers, C. D.: Inverse methods for atmospheric sounding: theory and practice, World Scientific Computing Co, 2000.

Todd, L. and Ramachandran, G.: Evaluation of algorithms for tomographic reconstruction of chemical concentrations in indoor air, Am. Ind. Hyg. Assoc. J., 55, 403-416, 1994.

Van der Sluis, A. and van der Vorst, H. A.: Numerical solution of large, sparse linear algebraic systems arising from tomography, in Seismic tomography: With application in global seismology and exploration geophysics, edited by Nolet, G., 10, 49-83, Reidel Publishing Company, Dordrecht, 1987.
Veitel, H., Kromer, B., Mößner, M., and Platt, U.: New techniques for measurements of atmospheric vertical trace gas profiles using DOAS, Environmental Science and Pollution Research, special issue 4, 17-26, 2002.

Vogel, B., Corsmeier, U., Vogel, H., Fiedler, F., Kühlwein, J., Friedrich, R., Obermeier, A., Weppner, J., Kalthoff, N., Bäumer, D., Bitzer, A., and Jay, K.: 2000: Comparison of measured and calculated motorway emission data, J. Atmos. Environ., 34, 2437-2450, 2000.

Wolfe, D. C.: On the application optical computed tomography to remote air pollution measurements, Ph.D. thesis, Stanford University, Palo Alto, CA, 1980.

Yost, M. G., Gadgil, A. J., Drescher, A. C., Zhou, Y., Simonds, M. A., Levine, S. P., Nazaroff, W., and Saisan, P.: Imaging indoor tracer-gas concentrations with computed tomography: experimental results with a remote sensing ftir system, Am. Ind. Hyg. Ass. J., 55, 395-402, 1994.

Ziemann, A., Arnold, K., and Raabe, A.: Acoustic tomography as a method to identify small-scale land surface characteristics, acta acustica united with ACUSTICA, 87, 731-737, 2001. 